\title{
Copper-Catalyzed Annulation- Cyanotrifluoromethylation of 1,6-Enynes Toward 1-Indanones via a Radical Process
}

\author{
Tian-Shu Zhang ${ }^{1}$, Wen-Juan Hao ${ }^{2}$, Pei-Jun Cai ${ }^{1 *}$, Guigen $\mathrm{Li}^{3,4}$, Shu-Jiang $T u^{2}$ and \\ Bo Jiang ${ }^{2 *}$ \\ ${ }^{1}$ School of Chemical Engineering \& Technology, China University of Mining and Technology, Xuzhou, China, ${ }^{2}$ Jiangsu Key \\ Laboratory of Green Synthetic Chemistry for Functional Materials, School of Chemistry \& Materials Science, Jiangsu Normal \\ University, Xuzhou, China, ${ }^{3}$ Collaborative Innovation Center of Chemistry for Life Sciences, Institute of Chemistry and \\ BioMedical Sciences, Nanjing University, Nanjing, China, ${ }^{4}$ Department of Chemistry and Biochemistry, Texas Tech University, \\ Lubbock, TX, United States
}

OPEN ACCESS

Edited by:

Nam-Jung Kim,

Kyung Hee University, South Korea

Reviewed by:

Albert Moyano,

University of Barcelona, Spain Alexandre Pradal,

UMR8232 Institut Parisien de Chimie

Moléculaire, France

Norio Shibata,

Nagoya Institute of Technology, Japan

${ }^{*}$ Correspondence:

Pei-Jun Cai

pjcai@cumt.edu.cn

Bo Jiang

jiangchem@jsnu.edu.cn

Specialty section: This article was submitted to

Organic Chemistry,

a section of the journal

Frontiers in Chemistry

Received: 13 January 2020 Accepted: 11 March 2020

Published: 17 April 2020

Citation:

Zhang T-S, Hao W-J, Cai P-J, Li G,

Tu S-J and Jiang B (2020)

Copper-Catalyzed

Annulation-Cyanotrifluoromethylation of 1,6-Enynes Toward 1-Indanones via

a Radical Process.

Front. Chem. 8:234

doi: 10.3389/fchem.2020.00234
A new Cu(II)-catalyzed annulation-cyanotrifluoromethylation of 1,6-enynes with Togni's reagent and trimethylsilyl cyanide (TMSCN) has been established, enabling the direct construction of trifluoromethylated 1-indanones with an all-carbon quaternary center in good yields. This reaction was performed by using low-cost $\mathrm{Cu}(\mathrm{OTf})_{2}$ as the catalyst and Togni's reagent as both the radical initiator and a $\mathrm{CF}_{3}$ source, providing an efficient protocol for building up an 1-indanone framework with wide functional group compatibility. The reaction mechanism was proposed through a radical triggered addition/5-exo-dig cyclization/oxidation/nucleophilic cascade.

Keywords: $\mathrm{Cu}(\mathrm{II})$ catalysis, annulation-difunctionalization, cyanotrifluoromethylation, 1,6-enynes, 1-indanones

\section{INTRODUCTION}

Trifluoromethylation of organic molecular skeletons has attracted considerable attention in pharmaceutical chemistry, agrochemicals, and functional materials, owing to the fact that incorporation of the trifluoromethyl group into organic molecules can modulate their abilities including lipophilicity, bioavailability, and metabolic stability (Umemoto, 1996; Müller et al., 2007; Hagmann, 2008; Studer, 2012; Yang et al., 2015). Therefore, many efforts have been done in the past few decades, which mainly depended on transition-metal-catalyzed trifluoromethylation reactions. Such reactions enable direct construction of the $\mathrm{C}_{-} \mathrm{CF}_{3}$ bond in an atom-economic manner and provide efficient and practical methods for the collection of trifluoromethyl-containing compounds, such as catalytic trifluoromethylation of alkane (Pan et al., 2011; Fu et al., 2012; Kuninobu et al., 2015; Wang et al., 2015; Xiao et al., 2019), alkenes (Chu and Qing, 2012; Shimizu et al., 2012; Zhu and Buchwald, 2013; Lin et al., 2016; He et al., 2018), and alkynes (Ge et al., 2014; Iqbal et al., 2014; Tomita et al., 2015; Wu et al., 2017; Huang et al., 2018). Among them, a vast majority of reports focused on the difunctionalization of alkenes or enynes (He et al., 2014a,b), such as hydrotrifluoromethylation (Wilger et al., 2013; Wu et al., 2013), carbotrifluoromethylation (Chen et al., 2013; Egami et al., 2013; Liu et al., 2013), and oxytrifluoromethylation (Egami et al., 2012; Janson et al., 2012; Li and Studer, 2012; Zhu and Buchwald, 2012) for their high utilization by incorporating trifluoromethyl groups into target molecules across the unsaturated $\pi$ system. On the other hand, 1-indanones are privileged structural motifs commonly present 


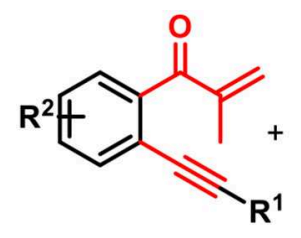<smiles>O=C1[OH+]C(C(F)(F)F)c2ccccc21</smiles><smiles>CC(C)(C)N</smiles>

\section{$\underset{\mathrm{K}_{3} \mathrm{PO}_{4}, \mathrm{CH}_{3} \mathrm{CN}}{\mathrm{Cu}(\mathrm{OTf})_{2} \text {, phen }}$ \\ Ar, $50^{\circ} \mathrm{C}$}

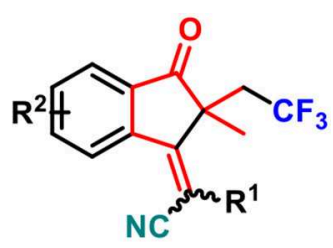

Graphical Abstract | A new Cu(II)-catalyzed annulation-cyanotrifluoromethylation of 1,6-enynes with Togni's reagent and TMSCN has been established, enabling the direct construction of trifluoromethylated 1-indanones with an all-carbon quaternary center in good yields. This reaction was performed by using low-cost $\mathrm{Cu}(\mathrm{OTf})_{2}$ as the catalyst and Togni's reagent as both the radical initiator and a CF3 source, providing an efficieA new Cu(II)-catalyzed annulation-cyanotrifluoromethylation of 1,6-enynes with Togni's reagent and TMSCN has been established, enabling the direct construction of trifluoromethylated 1-indanones with an all-carbon quaternary center in good yields. This reaction was performed by using low-cost $\mathrm{Cu}(\mathrm{OTf})_{2}$ as the catalyst and Togni's reagent as both the radical initiator and a $\mathrm{CF}_{3}$ source, providing an efficient protocol for building up 1-indanone framework with wide functional group compatibility. The reaction mechanism was proposed through a radical triggered addition/5-exo-dig cyclization/oxidation/nucleophilic cascade. nt protocol for building up 1-indanone framework with wide functional group compatibility. The reaction mechanism was proposed through a radical triggered addition/5-exo-dig cyclization/oxidation/nucleophilic cascade.

in many bioactive molecules and natural products such as Pterosin B and C (Nagle et al., 2000; Wessig and Teubner, 2006), pauciflorol F (Dai et al., 1998; Nitta et al., 2002; Ito et al., 2004), and (+)-indacrinone (DeSolms et al., 1978) (Figure 1). Consequently, many chemists made their contributions to establish numerous elegant protocols for their synthesis including Friedel-Crafts acylation (Koelsch, 1932; Frank et al., 1944), Grignard reactions (Bergmann, 1956; Manning et al., 1981), and transition metal-catalyzed annulation of arylalkynes (Shintani et al., 2007; Chernyak et al., 2011; He et al., 2018; Song et al., 2019), radical addition-cyclization of 1,6-enynes (Shen et al., 2018a,b, 2019), and other methods (Zhu et al., 2017, 2018a,b; Shi et al., 2019a). To the best of our knowledge, introduction of a trifluoromethyl group into the 1-indanone framework via a radical-triggered annulationdifunctionalization strategy remains elusive.

Multicomponent reactions (MCRs) represent an attractive and powerful tool for building complex molecular architectures under usually mild conditions (Hao et al., 2016; Wang et al., 2016a,b; Ji et al., 2019; Liu et al., 2019; Qin et al., 2019; Shi et al., 2019b). Radical-triggered annulation-difunctionalization cascades, standing at the intersection of both radical and multicomponent transformations, constitute a unique reaction category, which enables direct assembly of difunctionalized cyclic systems containing both isocyclic and heterocyclic skeletons which are not available from other methods. As a result, lots of unsaturated compounds endowed with alkene and/or alkyne units are devised and prepared as radical acceptors to capture the various radical species (Chen et al., 2008; Liu et al., 2014; Kong et al., 2015; Wang F. et al., 2016; Zhang et al., 2019). Generally, the success of the radical annulationdifunctionalization relies on the radical continuous transfer across the unsaturated systems through a synergistic orientation process. Over the years, our group has been heavily involved in the development of new annulation-difunctionalization cascades for multiple ring formations. For example, we reported a copper-catalyzed annulation-halofluoroalkylation of 1,6-enynes, leading to the atom-economic and highly stereoselective protocol toward functionalized 1-indenones (Scheme 1, path a) (Shen et al., 2019). To continue our interest in this project, we approach a radical addition-cyclization strategy to install

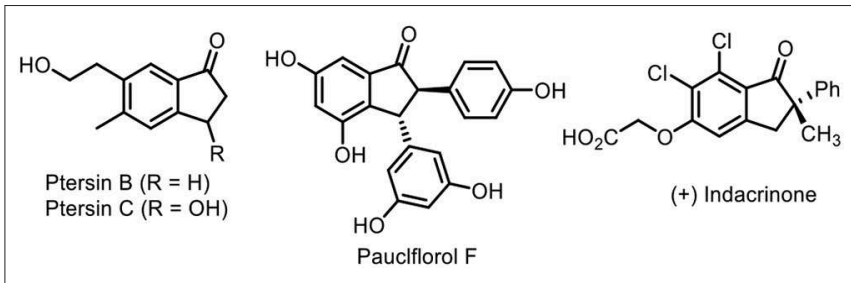

FIGURE 1 | 1-Indanone-containing natural products.

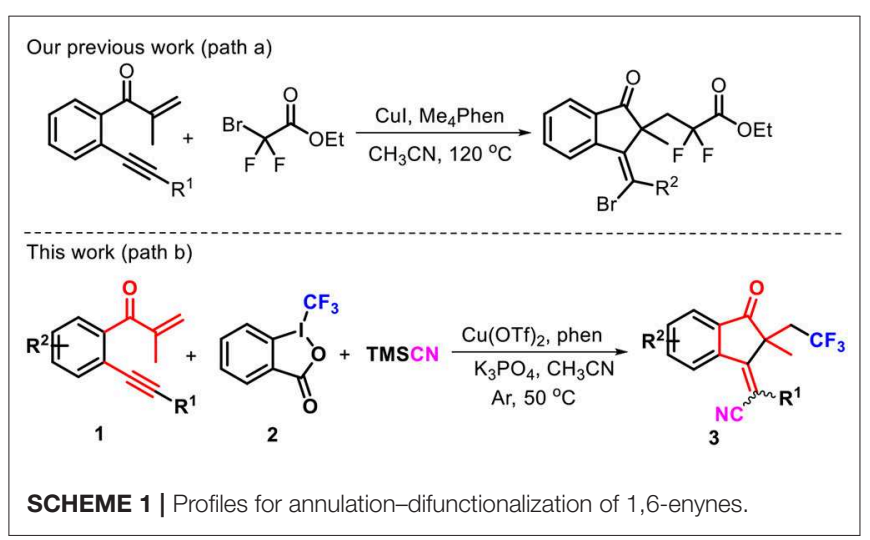

both trifluoromethyl and cyano moieties into the 1-indenone framework, due to the behaviors of trifluoromethyl and cyano groups in the wide application potentiality in assigning and discovering new biological lead compounds. An extensive literature survey revealed that the radical-triggered annulationcyanotrifluoromethylation of 1,6-enynes toward 1 -indanones remains unreported to date. For this reason, the coppercatalyzed annulation-cyanotrifluoromethylation of 1,6-enynes 1 with Togni's reagent $\mathbf{2 a}$ and trimethylsilyl cyanide (TMSCN) was carried out by 1,10-phenanthroline (phen) as the ligand, enabling a radical-induced three-component cascade to access trifluoromethylated 1-indanones 3 with generally good yields (Scheme 1, path b). Remarkably, some cases showed complete stereoselectivity, and only E-selectivity was observed. Herein, we report this copper-catalyzed radical transformation. 


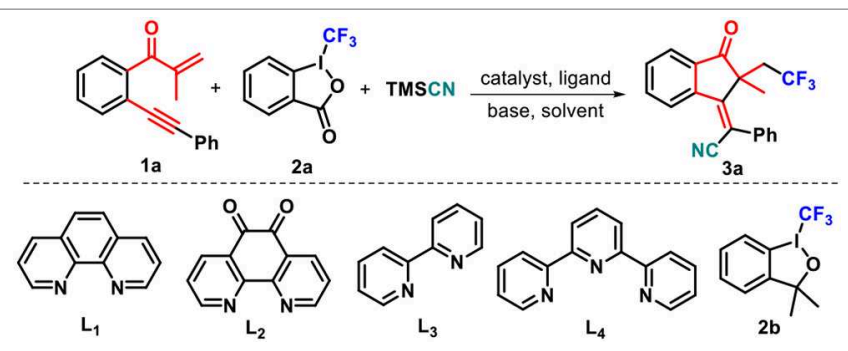

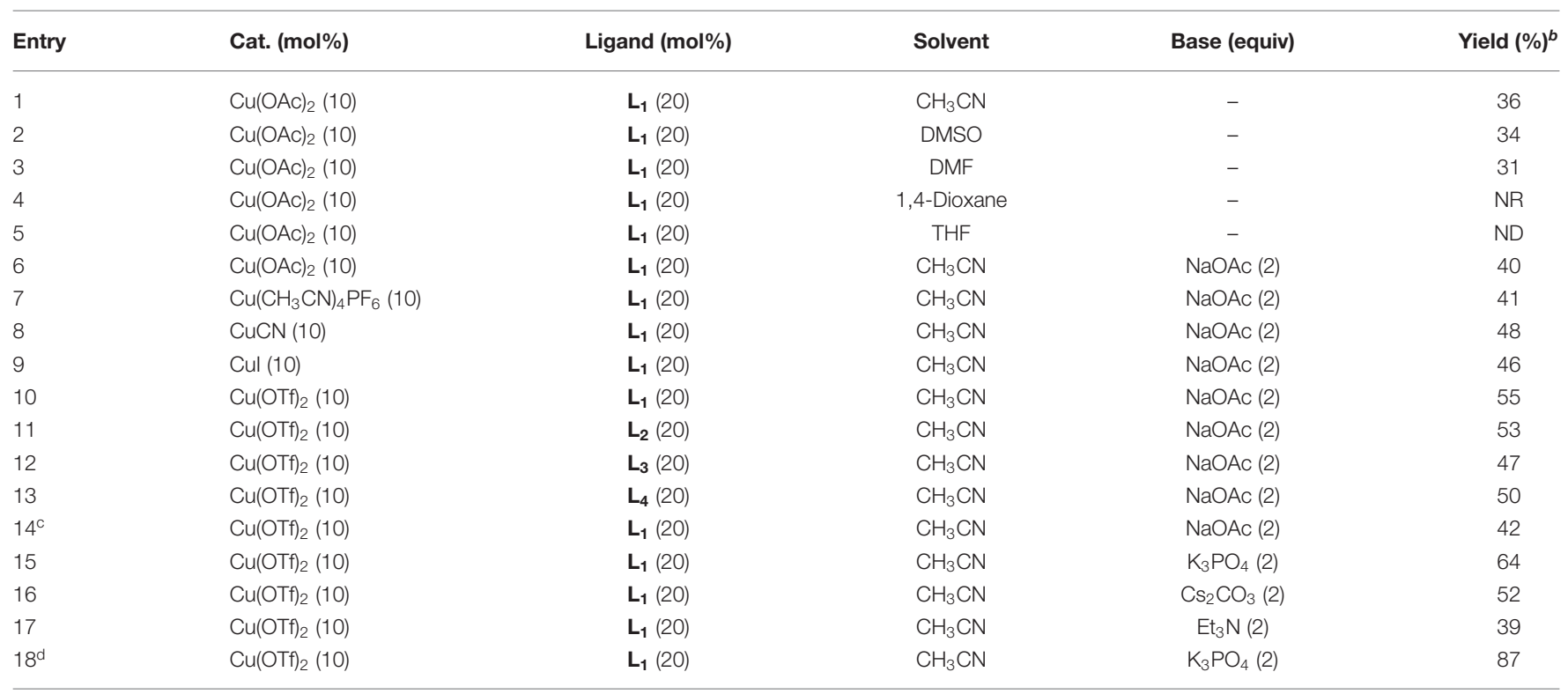

${ }^{[a]}$ Reaction conditions: $1(0.2 \mathrm{mmol}), 2(0.4 \mathrm{mmol}), \mathrm{Cu}(\mathrm{OTf})_{2}(10 \mathrm{~mol} \%), \mathbf{L 1}(20 \mathrm{~mol} \%), \mathrm{K}_{3} \mathrm{PO}_{4}(0.4 \mathrm{mmol})$, acetonitrile $(2.0 \mathrm{ml})$, TMSCN $(0.4 \mathrm{mmol})$, Ar conditions at $50^{\circ} \mathrm{C}$ for $3 \mathrm{~h}$. ${ }^{[b]}$ /solated yield based on substrates 1. ${ }^{[c]}$ Umemoto's reagent $\mathbf{2} \boldsymbol{b}$ was used. ${ }^{[d]}$ Mole ratio of $\mathbf{1 a}$, 2a, and TMSCN in 1:3:2.

\section{RESULTS AND DISCUSSION}

At the outset of our studies, we chose the preformed 1,6-enyne 1a, Togni's reagent $\mathbf{2 a}$, and TMSCN as the model substrate (Table 1). To our delight, the reaction of $\mathbf{1 a}$ with $\mathbf{2 a}$ and TMSCN in a $1: 2: 2 \mathrm{~mol}$ ratio catalyzed by $10 \mathrm{~mol} \% \mathrm{Cu}(\mathrm{OAc})_{2}$ proceeded smoothly in acetonitrile at $50^{\circ} \mathrm{C}$ by using 1,10 phenanthroline (phen, $\mathrm{L}_{1}$ ) as a ligand, and the target product $3 \mathbf{a}$ as a sole $(E)$-stereoisomer was obtained in $36 \%$ yield. The following screening of solvents showed that the use of DMSO and DMF led to a slightly decreased yield of $\mathbf{3 a}$ compared with acetonitrile (entries 2 and 3 vs. entry 1), whereas both 1,4dioxane and THF completely suppressed the formation of $\mathbf{3 a}$ (entries 4 and 5). Thus, acetonitrile was the best solvent for the reaction. An employment of $\mathrm{NaOAc}$ as the base facilitated the reaction process, delivering $40 \%$ yield of the desired product $\mathbf{3 a}$ (entry 6). After that, we conducted the screening of a variety of copper salts, such as $\mathrm{Cu}\left(\mathrm{CH}_{3} \mathrm{CN}\right)_{4} \mathrm{PF}_{6}, \mathrm{CuCN}, \mathrm{CuI}$, and $\mathrm{Cu}(\mathrm{OTf})_{2}$, that are often utilized in catalytic transformations, for this addition-cyclization cascade by using acetonitrile as the reaction medium. All these catalysts could promote the conversion of 1a into $\mathbf{3 a}$ (entries 7-10), and the latter one showed the best catalytic performance in the current reaction, generating product $3 \mathbf{a}$ in $55 \%$ yield (entry 10 ). As the next optimization step, several ligands, such as 1,10-phenanthroline-5,6-dione
$\left(\mathrm{L}_{2}\right), 2,2^{\prime}$-bipyridine $\left(\mathrm{L}_{3}\right)$, and $2,2^{\prime}: 6^{\prime}, 2^{\prime \prime}$-terpyridine $\left(\mathrm{L}_{4}\right)$, were investigated and anticipated to enhance the yield of product 3a. Disappointingly, ligands $\mathrm{L}_{2}-\mathrm{L}_{4}$ showed slightly weaker performance on the conversion of $\mathbf{1 a}$ into $\mathbf{3 a}$ as compared with $\mathrm{L}_{1}$ (entries 11-13). Using Togni's reagent $\mathbf{2 b}$ to replace 2a resulted in a lower conversion (42\%, entry 14 vs. entry 10$)$. Different bases such as potassium phosphate tribasic $\left(\mathrm{K}_{3} \mathrm{PO}_{4}\right)$, trimethylamine $\left(\mathrm{Et}_{3} \mathrm{~N}\right)$, and cesium carbonate $\left(\mathrm{Cs}_{2} \mathrm{CO}_{3}\right)$ were then screened. The results indicated that $\mathrm{K}_{3} \mathrm{PO}_{4}$ could improve the reaction, providing product $3 \mathbf{a}$ in $64 \%$ yield. After careful optimizations, we found that fine-tuning the substrate ratio 1a/2a/TMSCN to 1:3:2 delivered product $3 \mathbf{a}$ in a higher $87 \%$ yield (entry 18).

With the optimized conditions in hand (Table 1, entry 18 ), the substrate scope of this radical-triggered annulationcyanotrifluoromethylation of 1,6-enynes was investigated. The results were presented in Scheme 2. Upon repeating the reaction with $\mathbf{2 a}$ and TMSCN, substrate $\mathbf{1}$ with diverse substituents such as fluoro (1b), chloro (1c and 1d), and bromo (1e) groups on the arylalkynyl moiety all work well, giving the corresponding functionalized (E)-1-indanones $\mathbf{3 b}-\mathbf{3} \mathbf{e}$ in $45-$ $78 \%$ yields. Notably, substrates $\mathbf{1 c}-\mathbf{1 e}$ could completely orient the E-selectivity to the target products $\mathbf{3 c}-\mathbf{3 e}$. Alternatively, both cyclopropyl 1 ff and $n$-butyl $1 \mathrm{~g}$ counterparts were proven to be favorable, enabling radical-induced cyclization reactions 

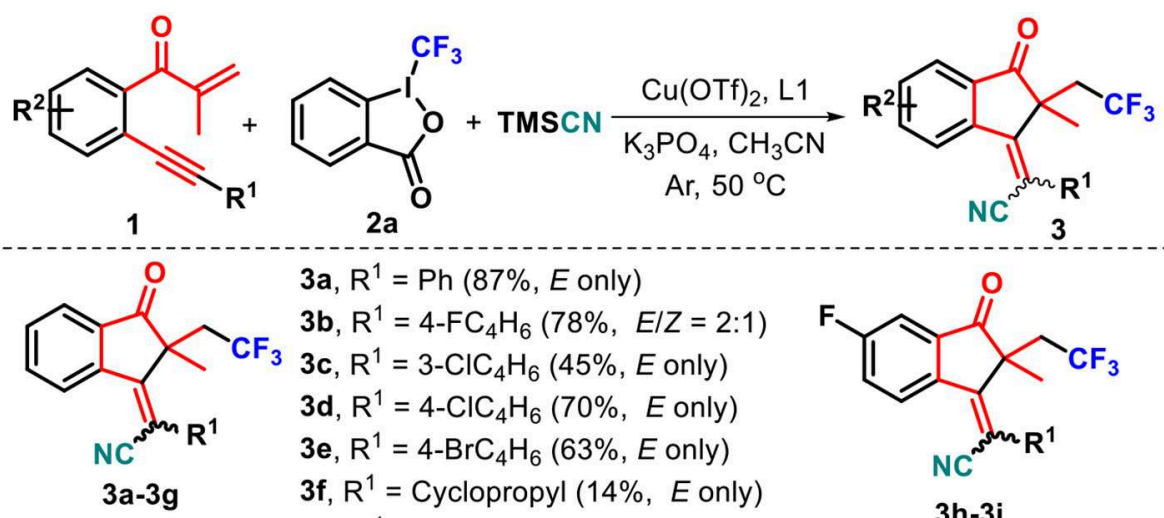

3a, $\mathrm{R}^{1}=\mathrm{Ph}(87 \%, E$ only)

$3 b, \mathrm{R}^{1}=4-\mathrm{FC}_{4} \mathrm{H}_{6}(78 \%, E / Z=2: 1)$

3c, $\mathrm{R}^{1}=3-\mathrm{ClC}_{4} \mathrm{H}_{6}(45 \%, E$ only)

$3 \mathrm{~d}, \mathrm{R}^{1}=4-\mathrm{ClC}_{4} \mathrm{H}_{6}(70 \%$, E only)

3e, $\mathrm{R}^{1}=4-\mathrm{BrC}_{4} \mathrm{H}_{6}$ (63\%, E only)

3f, $\mathrm{R}^{1}=$ Cyclopropyl (14\%, E only)

3g, $\mathrm{R}^{1}={ }^{n}$ Butyl $(30 \%, E$ only)<smiles>[R]C([N])=C1c2ccc(F)cc2C(=O)C1(C)CC(F)(F)F</smiles>

3h-3j<smiles>[R]C(C)=C1c2ccc(Cl)cc2C(=O)C1(C)CC(F)(F)F</smiles>

3k-3o

3k, $\mathrm{R}^{1}=\mathrm{Ph}(48 \%, E$ only)

3I, $\mathrm{R}^{1}=4-\mathrm{FC}_{4} \mathrm{H}_{6}(61 \%, E$ only $)$

$3 \mathrm{~m}, \mathrm{R}^{1}=p$-Tolyl $(62 \%, E / Z=2: 1)$

3n, $\mathrm{R}^{1}=4-\mathrm{EtC}_{4} \mathrm{H}_{6}(59 \%, E / Z=10: 3)$

3o, $\mathrm{R}^{1}=\operatorname{PMP}(69 \%, E / Z=5: 4)$<smiles>[R]C(C#N)=C1c2cc(C)ccc2C(=O)C1(C)CI</smiles>

$3 t-3 x$<smiles>COC(=O)C(C#N)=C1c2ccc(C)cc2C(=O)C1(C)CC(F)(F)F</smiles>

$3 p(65 \%, E$ only)<smiles>[R]C(C)=C1c2cc(Cl)ccc2C(=O)C1(C)CC(F)(F)F</smiles>

$3 r-3 s$

$3 \mathrm{~h}, \mathrm{R}^{1}=\mathrm{Ph}(42 \%, E / Z=2: 1)$

3i, $\mathrm{R}^{1}=p$-Tolyl $(55 \%, E / Z=5: 2)$

$3 \mathrm{j}, \mathrm{R}^{1}={ }^{-}{ }^{t} \mathrm{BuC}_{6} \mathrm{H}_{4}(64 \%, E / Z=5: 3)$<smiles>COc1ccc2c(c1)C(=O)C(C)(CCF)/C2=C(/C#N)c1ccc(Cl)cc1</smiles>

$3 q(64 \%, E$ only $)$

3t, $\mathrm{R}^{1}=\mathrm{Ph}(58 \%, E$ only)

$3 \mathrm{u}, \mathrm{R}^{1}=4-\mathrm{ClC}_{4} \mathrm{H}_{6}(85 \%, E / Z=5: 3)$

3v, $\mathrm{R}^{1}=4-\mathrm{BrC}_{4} \mathrm{H}_{6}(45 \%, E / Z=2: 1)$

$3 w, \mathrm{R}^{1}=p$-Tolyl $(73 \%, E / Z=2: 1)$

$3 \mathbf{x}, \mathrm{R}^{1}=\operatorname{PMP}(82 \%, E / Z=5: 2)$

$3 r, \mathrm{R}^{1}=\mathrm{Ph}(71 \%, E$ only)

3s, $\mathrm{R}^{1}=p$-Tolyl $(62 \%, E / Z=5: 3)$

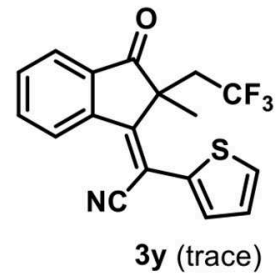

SCHEME 2 | Substrate scope of 1,6-enynes.

to offer the corresponding $(E)$-1-indanones $\mathbf{3 f}$ and $\mathbf{3 g}$ with complete stereoselectivities, albeit with low yields. Due to the pharmacological significance of fluorine-containing molecules compared to their non-fluorinated analogs, we decided to prepare 1,6-enynes $\mathbf{1 h}-\mathbf{1} \mathbf{j}$ containing the fluoro group residing in the 5-position of the internal arene ring and employed them to react with $\mathbf{2} \mathbf{a}$ and TMSCN. The reaction worked well, accessing the corresponding polyfluoro products $\mathbf{3 h}-\mathbf{3} \mathbf{j}$ in $42-64 \%$ yields and $5: 3$ to $5: 2 E / Z$ ratios. Other substituents including chloro $(\mathbf{1 k}-\mathbf{1 o}, \mathbf{1 r}$, and $\mathbf{1 s})$, methyl (1p and $\mathbf{1 t}-\mathbf{1 x})$, and methoxy (1q) located at the C4- or C5-position on the internal arene ring did not hamper this copper-catalyzed reaction, and a range of new substituted 1-indanones $\mathbf{3} \mathbf{k}-\mathbf{3} \mathbf{x}$ can be isolated in synthetically useful yields, in which a complete diastereoselectivity was also observed in the cases of $\mathbf{3 k}, \mathbf{3} \mathbf{l}, \mathbf{3 p}, \mathbf{3 q}, \mathbf{3 r}$, and $\mathbf{3 t}$. However, unsatisfactory $E / Z$ ratios were detected for the other products. Either electronically neutral $(\mathrm{H})$, poor (fluoro, chloro, and bromo), or rich [methyl, ethyl, $t$-butyl, and methoxy (PMP $=$ p-methoxyphenyl)] groups at the para-position of the arylalkynyl moiety $\left(\mathrm{R}^{1}\right)$ are well-tolerated with the catalytic conditions. Unfortunately, 1,6-enyne 1y carrying a 2-thienyl group was an ineffective reaction partner in this transformation. The structures of these resulting 1-indanones were fully characterized by NMR spectroscopy and HRMS data (Data Sheet 1).

To gain mechanistic insight into this transformation, radical trapping experiments were performed. When 2,2,6,6tetramethylpiperidine-1-oxyl (TEMPO) as a radical scavenger was subjected to the reaction conditions, the generation of 3a was completely suppressed (Scheme 3a). Similarly, BHT could inhibit the formation of $\mathbf{3 a}$. These results showed that the reaction may include a radical process. Moreover, the developed transformation could be valorized through postfunctionalization of indanone $\mathbf{3 q}$ (Scheme $\mathbf{3 b}$ ). The combination of $\mathrm{NaBH}_{4}$ and $\mathrm{I}_{2}$ was found to be effective to reduce $\mathbf{3 q}$ to give 2,3-dihydro- $1 H$-inden-1-ol 4 (51\% yield) (He et al., 2015; Chen et al., 2018). 
A Control experiments

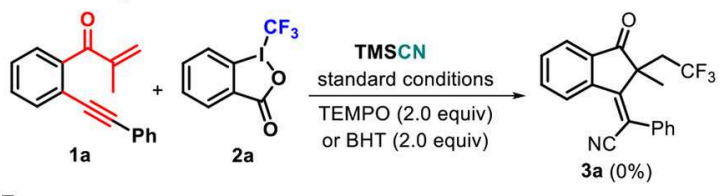

B Application of compound $3 q$

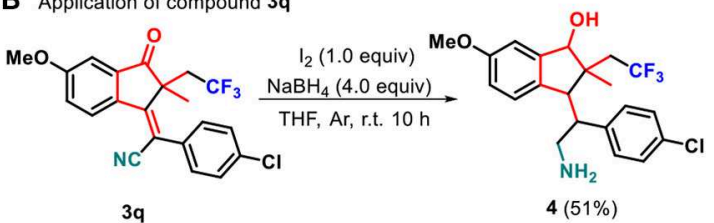

SCHEME 3 | Control experiments and application of compound 3q.

\section{MECHANISM}

According to these results and related literature (Kamigata et al., 1990; Liu et al., 2012; Pair et al., 2013; Yasu et al., 2013; He et al., 2014b; Shen et al., 2019), a plausible mechanism was proposed (Scheme 4). The copper(II) catalyst activates Togni's reagent $\mathbf{2 a}$ to give the activated complex $\mathbf{A}$, which releases a $\mathrm{Cu}$ (III) species and the trifluoromethyl radical. The latter rapidly adds to 1,6-enyne $\mathbf{1 a}$ to give the radical intermediate $\mathbf{B}$. In the presence of ligands and TMSCN, Cu(III) species activates an alkyne unit of intermediate $\mathbf{B}$ to drive 5-exo-dig cyclization, giving favorable anti-Cu(III) species C (Shen et al., 2019), some of which is converted into syn-Cu(III) species $\mathbf{C}^{\prime}$, together with trimethylsilyl 2-iodobenzoate D. Finally, anti-Cu(III) species C undergoes reductive elimination to give the desired $(E)$-product $3 \mathbf{a}$ as a major isomer and regenerate a $\mathrm{Cu}$ (II) complex to complete a catalytic cycle through the release of ligands (He et al., 2014b), whereas syn-Cu(III) species $\mathbf{C}^{\prime}$ undergoes the same reductive elimination to access minor $(Z)$-product $\mathbf{3 a}$.

\section{CONCLUSION}

In summary, we have established a copper-catalyzed annulationcyanotrifluoromethylation of 1,6-enynes with easily available Togni's reagent and TMSCN, by which a wide range of 1indanones with a quaternary carbon center were stereoselectively synthesized in generally good yields. Notably, a complete stereoselectivity could be detected in most cases. This approach is efficiently induced by Togni's reagent as a radical donor and ultimately terminated by TMSCN as the nucleophilic reagent. The transformation offered a new entry to prepare the $\mathrm{CF}_{3}$ containing 1-indanone skeleton via a complex radical additioncyclization cascade. Further investigations into the mechanism and its application will be conducted in due course.

\section{MATERIALS AND METHODS}

\section{General}

${ }^{1} \mathrm{H}$ NMR $\left({ }^{13} \mathrm{C}\right.$ NMR, ${ }^{19} \mathrm{~F}$ NMR) spectra were measured on a Bruker DPX $400-\mathrm{MHz}$ spectrometer in $\mathrm{CDCl}_{3}$ (DMSO- $d_{6}$ ) with chemical shift $(\delta)$ given in ppm relative to TMS as internal standard $[(\mathrm{s}=$ singlet, $\mathrm{d}=$ doublet, $\mathrm{t}=$ triplet, $\mathrm{brs}$

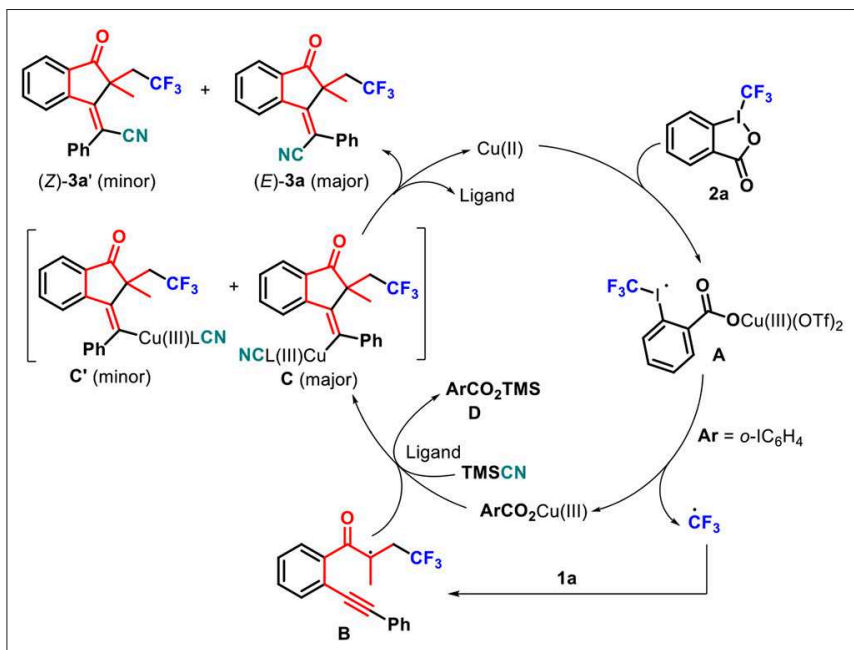

SCHEME 4 | Proposed reaction mechanism

= broad singlet, $\mathrm{m}=$ multiplet), coupling constant $(\mathrm{Hz})$ ]. HRMS (ESI) was done by using a micrOTOF-Q II HRMS/MS instrument (Bruker).

\section{General Procedure for the Synthesis of 3 Example for the Synthesis of 3a}

Under Ar conditions, a mixture of Togni's reagent $2(0.6 \mathrm{mmol})$, $\mathrm{Cu}(\mathrm{OTf})_{2}(0.02 \mathrm{mmol}), \mathrm{K}_{3} \mathrm{PO}_{4}(0.4 \mathrm{mmol})$, and ligand $\mathrm{L} 1(0.04$ $\mathrm{mmol}$ ) was added in a Schlenk tube. Acetonitrile was added into the tube. Then, 2-methyl-1-[2-(phenylethynyl)phenyl]prop-2en-1-one 1a $(0.2 \mathrm{mmol})$ and TMSCN $(0.4 \mathrm{mmol})$ were put in the system, stirred for $3 \mathrm{~h}$ at $50^{\circ} \mathrm{C}$ until thin-layer chromatography (TLC) revealed that conversion of the starting material 1a was complete. Next, the reaction mixture was concentrated in vacuum, and the resulting residue was purified by silica gel column chromatography (petroleum ether/ethyl acetate $=25: 1$, $\mathrm{v} / \mathrm{v}$ ) to afford the desired product $3 \mathbf{a}$.

\section{General Procedure for the Synthesis of 4}

Under Ar conditions, 3q (0.05 mmol), $\mathrm{NaBH}_{4}$ (3.0 equiv), and $\mathrm{I}_{2}$ (1.0 equiv) were added in a Schlenk tube. THF was added, and the reaction mixture was stirred at room temperature for $10 \mathrm{~h}$. The solution was treated with water and extracted with dichloromethane. The combined organic layers were washed with brine, dried over $\mathrm{Na}_{2} \mathrm{SO}_{4}$, concentrated in vacuum, and purified by preparative TLC (petroleum ether/ethyl acetate $=$ 2/1) to afford product 4 (He et al., 2015; Chen et al., 2018).

\section{(E)-2-[2-Methyl-3-oxo-2-(2,2,2-trifluoroethyl)-2,3-} dihydro- $1 \mathrm{H}$-inden-1-ylidene]-2-phenylacetonitrile (3a) Light yellow solid, $59 \mathrm{mg}$, 87\% yield; $\mathrm{mp} 105.2-106.1^{\circ} \mathrm{C} .{ }^{1} \mathrm{H} \mathrm{NMR}$ (400 MHz, $\left.\mathrm{CDCl}_{3} ; \delta, \mathrm{ppm}\right): 8.96(\mathrm{~d}, J=8.0 \mathrm{~Hz}, 1 \mathrm{H}), 7.94(\mathrm{~d}, J=$ $7.6 \mathrm{~Hz}, 1 \mathrm{H}), 7.90-7.85(\mathrm{~m}, 1 \mathrm{H}), 7.73-7.67(\mathrm{~m}, 1 \mathrm{H}), 7.54-7.49(\mathrm{~m}$, $3 \mathrm{H}), 7.46-7.41(\mathrm{~m}, 2 \mathrm{H}), 2.66-2.53(\mathrm{~m}, 1 \mathrm{H}), 2.25-2.12(\mathrm{~m}, 1 \mathrm{H})$, 1.19 (s, 3H). ${ }^{13} \mathrm{C}$ NMR (100 MHz, $\left.\mathrm{CDCl}_{3} ; \delta, \mathrm{ppm}\right): 202.3,153.2$, $144.5,136.3,135.5,133.0,132.4,129.9,129.5,129.2,125.0(\mathrm{q}, J=$ $85.1 \mathrm{~Hz}, \mathrm{CF}_{3}$ ), 118.9, 109.1, 49.8, 40.4 (q, $J=27.6 \mathrm{~Hz}, \mathrm{CH}_{2} \mathrm{CF}_{3}$ ), 25.1. ${ }^{19} \mathrm{~F} \mathrm{NMR}\left(376 \mathrm{MHz}, \mathrm{CDCl}_{3} ; \delta, \mathrm{ppm}\right):-61.29$ (s, 3F). IR 
$\left(\mathrm{KBr}, v, \mathrm{~cm}^{-1}\right): 2,200,1,721,1,577,1,447,1,361,1,256,1,138,967$, 775. HRMS (ESI, $m / z$ ): calcd for $\mathrm{C}_{20} \mathrm{H}_{14} \mathrm{~F}_{3} \mathrm{NONa}[\mathrm{M}+\mathrm{Na}]^{+}$ 364.0919, found 364.0928.

(E)-2-(4-Fluorophenyl)-2-[2-methyl-3-oxo-2-(2,2,2trifluoroethyl)-2,3-dihydro- $1 \mathrm{H}$-inden-1ylidene]acetonitrile (3b, Major)

Light yellow solid, $56 \mathrm{mg}, 78 \%$ yield; $\mathrm{mp} 148.9-150.9^{\circ} \mathrm{C} .{ }^{1} \mathrm{H}$ NMR $\left(400 \mathrm{MHz}, \mathrm{CDCl}_{3} ; \delta, \mathrm{ppm}\right): 8.94(\mathrm{~d}, J=8.0 \mathrm{~Hz}, 1 \mathrm{H}), 7.94(\mathrm{~d}, J=$ $7.6 \mathrm{~Hz}, 1 \mathrm{H}), 7.86(\mathrm{~d}, J=8.0 \mathrm{~Hz}, 1 \mathrm{H}), 7.73-7.67(\mathrm{~m}, 1 \mathrm{H}), 7.46-7.42$ (m, 2H), 7.27-7.22 (m, 2H), 2.67-2.58 (m, 1H), 2.18-2.09 (m, $1 \mathrm{H}), 1.20$ (s, $3 \mathrm{H}) .{ }^{13} \mathrm{C}$ NMR $\left(100 \mathrm{MHz}, \mathrm{CDCl}_{3} ; \delta\right.$, ppm): 202.0, $163.3\left(\mathrm{~d},{ }^{1} \mathrm{~J}=247.0 \mathrm{~Hz}, \mathrm{CF}\right), 153.9,144.3,136.3,135.3,132.6$, $131.6\left(\mathrm{~d},{ }^{3} \mathrm{~J}=8.3 \mathrm{~Hz}, \mathrm{CF}\right), 128.9\left(\mathrm{~d},{ }^{4} J=3.7 \mathrm{~Hz}, \mathrm{CF}\right), 126.6,125.0$ $\left(\mathrm{q}, J=80.7 \mathrm{~Hz}, \mathrm{CF}_{3}\right), 117.4,117.2,116.4\left(\mathrm{~d},{ }^{2} J=21.8 \mathrm{~Hz}, \mathrm{CF}\right)$, $108.0,49.8,40.3\left(\mathrm{q}, J=27.8 \mathrm{~Hz}, \mathrm{CH}_{2} \mathrm{CF}_{3}\right), 25.2 .{ }^{19} \mathrm{~F}$ NMR $(376$ $\left.\mathrm{MHz}, \mathrm{CDCl}_{3} ; \delta, \mathrm{ppm}\right):-61.31$ (s, 3F), -109.82 (s, 1F). IR ( $\mathrm{KBr}$, $\left.v, \mathrm{~cm}^{-1}\right): 2,202,1,724,1,599,1,509,1,361,1,257,1,142,1,070$, 776. HRMS (ESI, $m / z$ ): calcd for $\mathrm{C}_{20} \mathrm{H}_{13} \mathrm{~F}_{4} \mathrm{NONa}[\mathrm{M}+\mathrm{Na}]^{+}$ 382.0825 , found 382.0784 .

(E)-2-(3-Chlorophenyl)-2-[2-Methyl-3-oxo-2-(2,2,2-

Trifluoroethyl)-2,3-Dihydro-1 $H$-Inden-1Ylidene]Acetonitril (3c)

Light yellow solid, $34 \mathrm{mg}, 45 \%$ yield; $\mathrm{mp} 174.6-177.1^{\circ} \mathrm{C} .{ }^{1} \mathrm{H}$ NMR $\left(400 \mathrm{MHz}, \mathrm{CDCl}_{3} ; \delta, \mathrm{ppm}\right): 8.94(\mathrm{~d}, J=8.0 \mathrm{~Hz}, 1 \mathrm{H}), 7.96(\mathrm{~d}$, $J=7.6 \mathrm{~Hz}, 1 \mathrm{H}), 7.91-7.86(\mathrm{~m}, 1 \mathrm{H}), 7.75-7.69(\mathrm{~m}, 1 \mathrm{H}), 7.52-$ $7.42(\mathrm{~m}, 3 \mathrm{H}), 7.34(\mathrm{~d}, J=7.2 \mathrm{~Hz}, 1 \mathrm{H}), 2.69-2.61(\mathrm{~m}, 1 \mathrm{H}), 2.21-$ $2.12(\mathrm{~m}, 1 \mathrm{H}), 1.21(\mathrm{~s}, 3 \mathrm{H}) .{ }^{13} \mathrm{C}$ NMR $\left(100 \mathrm{MHz}, \mathrm{CDCl}_{3} ; \delta\right.$, ppm): 201.9, 154.0, 144.2, 136.4, 135.6, 135.2, 134.6, 132.7, 130.5 . $130.2,129.8,127.7,125.1\left(\mathrm{q}, J=78.5 \mathrm{~Hz}, \mathrm{CF}_{3}\right), 118.5,107.6$, 49.8, $40.2\left(\mathrm{q}, J=55.5 \mathrm{~Hz}, \mathrm{CH}_{2} \mathrm{CF}_{3}\right), 25.2 .{ }^{19} \mathrm{~F}$ NMR $(376 \mathrm{MHz}$, $\left.\mathrm{CDCl}_{3} ; \delta, \mathrm{ppm}\right):-61.31$ (s, 3F). IR $\left(\mathrm{KBr}, v, \mathrm{~cm}^{-1}\right): 2,204,1,728$, $1,595,1,336,1,260,1,140,1,069,776$. HRMS (ESI, $m / z)$ ): calcd for $\mathrm{C}_{20} \mathrm{H}_{13} \mathrm{ClF}_{3} \mathrm{NONa}[\mathrm{M}+\mathrm{Na}]^{+}$398.0529, found 398.0520.

(E)-2-(4-Chlorophenyl)-2-[2-Methyl-3-oxo-2-(2,2,2Trifluoroethyl)-2,3-Dihydro-1H-Inden-1ylidene]Acetonitrile (3d)

Light yellow solid, $53 \mathrm{mg}, 70 \%$ yield; $\mathrm{mp} 154.5-155.3^{\circ} \mathrm{C} .{ }^{1} \mathrm{H}$ NMR $\left(400 \mathrm{MHz}, \mathrm{CDCl}_{3} ; \delta, \mathrm{ppm}\right): 8.94(\mathrm{~d}, J=8.4 \mathrm{~Hz}, 1 \mathrm{H}), 7.95(\mathrm{~d}, J$ $=7.6 \mathrm{~Hz}, 1 \mathrm{H}), 7.91-7.85(\mathrm{~m}, 1 \mathrm{H}), 7.75-7.69(\mathrm{~m}, 1 \mathrm{H}), 7.50(\mathrm{~d}$, $J=8.4 \mathrm{~Hz}, 2 \mathrm{H}), 7.39(\mathrm{~d}, J=8.4 \mathrm{~Hz}, 2 \mathrm{H}), 2.69-2.59(\mathrm{~m}, 1 \mathrm{H})$, 2.20-2.11 (m, 1H), $1.20(\mathrm{~s}, 3 \mathrm{H}) .{ }^{13} \mathrm{C}$ NMR $\left(100 \mathrm{MHz}, \mathrm{CDCl}_{3} ; \delta\right.$, ppm): 201.9, 153.9, 144.2, 136.4, 136.3, 135.6, 132.7, 131.4, 131.0, $129.6,125.1\left(\mathrm{q}, J=78.5 \mathrm{~Hz}, \mathrm{CF}_{3}\right), 118.6,107.8,49.8,40.7(\mathrm{q}, J=$ $\left.27.7 \mathrm{~Hz}, \mathrm{CH}_{2} \mathrm{CF}_{3}\right), 25.2 .{ }^{19} \mathrm{~F}$ NMR $\left(376 \mathrm{MHz}, \mathrm{CDCl}_{3} ; \delta, \mathrm{ppm}\right)$ : -61.28 (s, 3F). IR ( $\left.\mathrm{KBr}, v, \mathrm{~cm}^{-1}\right): 2,204,1,729,1,593,1,491$, $1,360,1,256,1,143,1,072,835,776$. HRMS (ESI, $m / z$ ): calcd for $\mathrm{C}_{20} \mathrm{H}_{13} \mathrm{ClF}_{3} \mathrm{NONa}[\mathrm{M}+\mathrm{Na}]^{+}$398.0529, found 398.0569.

(E)-2-(4-Bromophenyl)-2-[2-Methyl-3-oxo-2-(2,2,2Trifluoroethyl)-2,3-dihydro- $1 \mathrm{H}$-inden-1ylidene]Acetonitrile (3e)

Light yellow solid, $53 \mathrm{mg}, 63 \%$ yield; $\mathrm{mp} 103.9-104.7^{\circ} \mathrm{C} .{ }^{1} \mathrm{H}$ NMR $\left(400 \mathrm{MHz}, \mathrm{CDCl}_{3} ; \delta, \mathrm{ppm}\right): 8.93(\mathrm{~d}, J=8.0 \mathrm{~Hz}, 1 \mathrm{H}), 7.95(\mathrm{~d}, J$ $=7.6 \mathrm{~Hz}, 1 \mathrm{H}), 7.91-7.85(\mathrm{~m}, 1 \mathrm{H}), 7.74-7.68(\mathrm{~m}, 1 \mathrm{H}), 7.66(\mathrm{~d}$, $J=8.4 \mathrm{~Hz}, 2 \mathrm{H}), 7.32(\mathrm{~d}, J=8.4 \mathrm{~Hz}, 2 \mathrm{H}), 2.71-2.59(\mathrm{~m}, 1 \mathrm{H})$,
2.21-2.11 (m, 1H), $1.20(\mathrm{~s}, 3 \mathrm{H}) .{ }^{13} \mathrm{C}$ NMR (100 MHz, $\mathrm{CDCl}_{3}$; $\delta$, ppm): 201.9, 153.9, 144.2, 136.4, 135.6, 132.7, 132.5, 131.9, $131.2,125.1\left(\mathrm{q}, J=77.7 \mathrm{~Hz}, \mathrm{CF}_{3}\right), 124.5,118.5,107.8,49.8,40.4$ $\left(\mathrm{q}, J=27.8 \mathrm{~Hz}, \mathrm{CH}_{2} \mathrm{CF}_{3}\right), 25.2 .{ }^{19} \mathrm{~F}$ NMR $\left(376 \mathrm{MHz}, \mathrm{CDCl}_{3}\right.$; $\delta$, ppm): -61.27 (s, 3F). IR $\left(\mathrm{KBr}, v, \mathrm{~cm}^{-1}\right): 2,205,1,728,1,585$, $1,486,1,360,1,255,1,142,1,069,1,011,968,832,723$. HRMS (ESI, $m / z$ ): calcd for $\mathrm{C}_{20} \mathrm{H}_{13} \mathrm{BrF}_{3} \mathrm{NONa}[\mathrm{M}+\mathrm{Na}]^{+} 442.0024$, found 442.0020 .

(E)-2-Cyclopropyl-2-[2-Methyl-3-oxo-2-(2,2,2trifluoroethyl)-2,3-dihydro- $1 \mathrm{H}$-inden-1ylidene]Acetonitrile (3f)

Light yellow oil, $9 \mathrm{mg}, 14 \%$ yield. ${ }^{1} \mathrm{H}$ NMR $\left(400 \mathrm{MHz}, \mathrm{CDCl}_{3} ; \delta\right.$, ppm): $7.82(\mathrm{~d}, J=7.6 \mathrm{~Hz}, 1 \mathrm{H}), 7.72-7.61(\mathrm{~m}, 2 \mathrm{H}), 7.46-7.39(\mathrm{~m}$, $1 \mathrm{H}), 5.99-5.89(\mathrm{~m}, 1 \mathrm{H}), 2.86-2.77(\mathrm{~m}, 1 \mathrm{H}), 2.65-2.58(\mathrm{~m}, 1 \mathrm{H})$, 2.58-2.46 (m, 4H), 1.33 (s, 3H). ${ }^{13} \mathrm{C}$ NMR $\left(100 \mathrm{MHz}, \mathrm{CDCl}_{3}\right.$; $\delta$, ppm): 203.5, 146.2, 135.9, 129.2, $124.4\left(\mathrm{q}, J=28.8 \mathrm{~Hz}, \mathrm{CF}_{3}\right)$, $118.9,110.0,97.0,48.1,40.0\left(\mathrm{q}, J=28.1 \mathrm{~Hz}, \mathrm{CH}_{2} \mathrm{CF}_{3}\right), 25.3,25.2$, 17.1. ${ }^{19} \mathrm{~F}$ NMR $\left(376 \mathrm{MHz}, \mathrm{CDCl}_{3} ; \delta\right.$, ppm): -61.78 (s, 3F). IR $\left(\mathrm{KBr}, v, \mathrm{~cm}^{-1}\right): 2,248,1,964,1,719,1,602,1,471,1,362,1,261$, $1,142,1,069,799$. HRMS (ESI, $m / z$ ): calcd for $\mathrm{C}_{17} \mathrm{H}_{14} \mathrm{~F}_{3} \mathrm{NONa}$ $[\mathrm{M}+\mathrm{Na}]^{+}$328.0920, found 328.0856.

\section{(E)-2-[2-Methyl-3-oxo-2-(2,2,2-Trifluoroethyl)-2,3-} dihydro- $1 \mathrm{H}$-inden-1-ylidene]hexanenitrile $(3 \mathrm{~g})$ Light yellow oil, $19 \mathrm{mg}, 30 \%$ yield. ${ }^{1} \mathrm{H}$ NMR $\left(400 \mathrm{MHz}, \mathrm{CDCl}_{3}\right.$; $\delta$, ppm): $8.88(\mathrm{~d}, J=8.0 \mathrm{~Hz}, 1 \mathrm{H}), 7.90(\mathrm{~d}, J=7.6 \mathrm{~Hz}, 1 \mathrm{H}), 7.83-$ $7.77(\mathrm{~m}, 1 \mathrm{H}), 7.64-7.59(\mathrm{~m}, 1 \mathrm{H}), 3.08-2.99(\mathrm{~m}, 1 \mathrm{H}), 2.82-2.72$ (m, $1 \mathrm{H}), 2.57-2.48(\mathrm{~m}, 2 \mathrm{H}), 1.83-1.69(\mathrm{~m}, 2 \mathrm{H}), 1.49-1.45(\mathrm{~m}$, $2 \mathrm{H}), 1.41(\mathrm{~s}, 3 \mathrm{H}), 1.01(\mathrm{t}, J=7.2 \mathrm{~Hz}, 3 \mathrm{H}) .{ }^{13} \mathrm{C}$ NMR $(100 \mathrm{MHz}$, $\left.\mathrm{CDCl}_{3} ; \delta, \mathrm{ppm}\right): 202.7,149.9,144.8,136.2,134.8,131.6,124.8$ (q, $\left.J=49.1 \mathrm{~Hz}, \mathrm{CF}_{3}\right), 118.8,110.3,49.2,40.3(\mathrm{q}, J=25.2 \mathrm{~Hz}$, $\left.\mathrm{CH}_{2} \mathrm{CF}_{3}\right), 31.8,30.0,23.7,22.6,13.9 .{ }^{19} \mathrm{~F} \mathrm{NMR}\left(376 \mathrm{MHz}, \mathrm{CDCl}_{3}\right.$; $\delta$, ppm): -62.09 (s, 3F). IR (KBr, $\left.v, \mathrm{~cm}^{-1}\right): 2,210,1,731,1,596$, $1,469,1,365,1,257,1,142,1,070$, 777. HRMS (ESI, $m / z$ ): calcd for $\mathrm{C}_{18} \mathrm{H}_{18} \mathrm{~F}_{3} \mathrm{NONa}[\mathrm{M}+\mathrm{Na}]^{+}$344.1213, found 344.1197.

\section{(E)-2-[5-Fluoro-2-methyl-3-oxo-2-(2,2,2-} trifluoroethyl)-2,3-dihydro- $1 \mathrm{H}$-inden-1-ylidene]-2phenylacetonitrile (3h, Major)

Light yellow solid, $30 \mathrm{mg}, 42 \%$ yield; $\mathrm{mp} 107.0-109.0^{\circ} \mathrm{C} .{ }^{1} \mathrm{H}$ NMR (400 MHz, $\left.\mathrm{CDCl}_{3} ; \delta, \mathrm{ppm}\right)$ : 9.00-8.94 (m, 1H), 7.59-7.55 (m, $2 \mathrm{H}), 7.51-7.48(\mathrm{~m}, 3 \mathrm{H}), 7.44-7.42(\mathrm{~m}, 2 \mathrm{H}), 2.62-2.53(\mathrm{~m}, 1 \mathrm{H})$, 2.24-2.15 (m, $1 \mathrm{H}), 1.19$ (s, 3H). ${ }^{13} \mathrm{C}$ NMR $\left(100 \mathrm{MHz}, \mathrm{CDCl}_{3} ; \delta\right.$, ppm): 201.3, $165.0\left(\mathrm{~d},{ }^{1} J=256.3 \mathrm{~Hz}, \mathrm{CF}\right), 152.1,140.6\left(\mathrm{~d},{ }^{5} J=\right.$ $2.5 \mathrm{~Hz}, \mathrm{CF}), 132.7,130.1,129.2,127.8\left(\mathrm{~d},{ }^{4} J=8.6 \mathrm{~Hz}, \mathrm{CF}\right), 126.3$, $123.5,123.3\left(\mathrm{~d},{ }^{2} J=26.8 \mathrm{~Hz}, \mathrm{CF}\right), 118.9,110.6\left(\mathrm{~d},{ }^{3} \mathrm{~J}=22.2 \mathrm{~Hz}\right.$, $\mathrm{CF}), 50.4,40.5\left(\mathrm{q}, J=27.8 \mathrm{~Hz}, \mathrm{CH}_{2} \mathrm{CF}_{3}\right), 25.1 .{ }^{19} \mathrm{~F}$ NMR (376 $\left.\mathrm{MHz}, \mathrm{CDCl}_{3} ; \delta, \mathrm{ppm}\right):-61.30$ (s, 3F), -104.65 (s, $\left.1 \mathrm{~F}\right)$. IR (KBr, $v$, $\left.\mathrm{cm}^{-1}\right): 2,205,1,732,1,600,1,488,1,362,1,257,1,186,1,141,1,067$, 949, 833. HRMS (ESI, $m / z$ ): calcd for $\mathrm{C}_{20} \mathrm{H}_{13} \mathrm{~F}_{4} \mathrm{NONa}[\mathrm{M}+\mathrm{Na}]^{+}$ 382.0825 , found 382.0832 .

(E)-2[(5-Fluoro-2-methyl-3-oxo-2-(2,2,2trifluoroethyl)-2,3-dihydro-1 $H$-inden-1-ylidene]-2-( $p$ tolyl)acetonitrile (3i, Major)

Light yellow solid, $41 \mathrm{mg}$, 55\% yield; $\mathrm{mp} 1,041-105.8^{\circ} \mathrm{C} .{ }^{1} \mathrm{H}$ NMR (400 MHz, $\left.\mathrm{CDCl}_{3} ; \delta, \mathrm{ppm}\right)$ : 8.98-8.93 (m, 1H), 7.58-7.52 (m, 
2H), 7.35-7.30 (m, 4H), 2.62-2.52 (m, 1H), 2.43 (s, 3H), 2.27$2.18(\mathrm{~m}, 1 \mathrm{H}), 1.20(\mathrm{~s}, 3 \mathrm{H}) .{ }^{13} \mathrm{C} \mathrm{NMR}\left(100 \mathrm{MHz}, \mathrm{CDCl}_{3} ; \delta, \mathrm{ppm}\right)$ : $201.5,164.9\left(\mathrm{~d},{ }^{1} J=256.2 \mathrm{~Hz}, \mathrm{CF}\right), 152.0,140.2,130.8,129.9$, $129.3,127.7\left(\mathrm{~d},{ }^{4} \mathrm{~J}=8.6 \mathrm{~Hz}, \mathrm{CF}\right), 126.3,123.5,123.2\left(\mathrm{~d},{ }^{2} \mathrm{~J}=\right.$ $23.6 \mathrm{~Hz}, \mathrm{CF}$ ), 119.0, 110.5 (d, $\left.{ }^{3} J=22.2 \mathrm{~Hz}, \mathrm{CF}\right), 50.4,40.4$ (q, $J$ $\left.=27.7 \mathrm{~Hz}, \mathrm{CH}_{2} \mathrm{CF}_{3}\right), 25.1,21.4 .{ }^{19} \mathrm{~F} \mathrm{NMR}\left(376 \mathrm{MHz}, \mathrm{CDCl}_{3} ; \delta\right.$, ppm): -61.29 (s, 3F), -104.91 (s, 1F). IR (KBr, $\left.v, \mathrm{~cm}^{-1}\right): 2,201$, $1,729,1,596,1,447,1,361,1,256,1,178,1,138,1,069,967,775$, 712. HRMS (ESI, $m / z$ ): calcd for $\mathrm{C}_{21} \mathrm{H}_{15} \mathrm{~F}_{4} \mathrm{NONa}[\mathrm{M}+\mathrm{Na}]^{+}$ 396.0982, found 396.0956 .

(E)-2-[4-(tert-Butyl)phenyl]-2-[5-fluoro-2-methyl-3oxo-2-(2,2,2-trifluoroethyl)-2,3-dihydro-1H-inden-1ylidene]acetonitrile (3j, Major)

Light yellow oil, $53 \mathrm{mg}$, 64\% yield. ${ }^{1} \mathrm{H} \mathrm{NMR}\left(400 \mathrm{MHz}, \mathrm{CDCl}_{3}\right.$; $\delta, \mathrm{ppm}):$ 8.99-8.92 (m, 1H), 7.53-7.48 (m, 3H), 7.36-7.32 (m, $3 \mathrm{H}), 2.63-2.53(\mathrm{~m}, 1 \mathrm{H}), 2.31-2.18(\mathrm{~m}, 1 \mathrm{H}), 1.37(\mathrm{~s}, 9 \mathrm{H}), 1.20(\mathrm{~s}$, $3 \mathrm{H}) .{ }^{13} \mathrm{C} \mathrm{NMR}\left(100 \mathrm{MHz}, \mathrm{CDCl}_{3} ; \delta, \mathrm{ppm}\right): 201.5,164.9\left(\mathrm{~d},{ }^{1} J\right.$ $=256.0 \mathrm{~Hz}, \mathrm{CF}), 153.4,140.8\left(\mathrm{~d},{ }^{5} \mathrm{~J}=2.6 \mathrm{~Hz}, \mathrm{CF}\right), 129.7,129.1$, 128.3, $127.7\left(\mathrm{~d},{ }^{4} J=8.5 \mathrm{~Hz}, \mathrm{CF}\right), 126.1,123.5,123.2\left(\mathrm{~d},{ }^{2} J=\right.$ $23.6 \mathrm{~Hz}, \mathrm{CF}), 119.0,110.5\left(\mathrm{~d},{ }^{3} J=22.2 \mathrm{~Hz}, \mathrm{CF}\right), 50.4,40.6$ (q, $J=$ $\left.27.7 \mathrm{~Hz}, \mathrm{CH}_{2} \mathrm{CF}_{3}\right), 34.9,31.3,25.0 .{ }^{19} \mathrm{~F} \mathrm{NMR}\left(376 \mathrm{MHz}, \mathrm{CDCl}_{3}\right.$; $\delta, \mathrm{ppm}):-61.26(\mathrm{~s}, 3 \mathrm{~F}),-104.94(\mathrm{~s}, 1 \mathrm{~F})$. IR $\left(\mathrm{KBr}, v, \mathrm{~cm}^{-1}\right)$ : 2,206, 1,734, 1,599, 1,487, 1,362, 1,258, 1,187, 1,141, 1,071, 949, 808. HRMS (ESI, $m / z$ ): calcd for $\mathrm{C}_{24} \mathrm{H}_{21} \mathrm{~F}_{4} \mathrm{NONa}[\mathrm{M}+\mathrm{Na}]^{+}$ 438.1451 , found 438.1458 .

\section{(E)-2-[5-Chloro-2-methyl-3-oxo-2-(2,2,2-} trifluoroethyl)-2,3-dihydro-1 $H$-inden-1-ylidene]-2phenylacetonitrile (3k)

Light yellow solid, $36 \mathrm{mg}$, $48 \%$ yield; $\mathrm{mp} 144.7-146.9^{\circ} \mathrm{C} .{ }^{1} \mathrm{H}$ NMR (400 MHz, $\left.\mathrm{CDCl}_{3} ; \delta, \mathrm{ppm}\right): 8.89(\mathrm{~d}, J=8.8 \mathrm{~Hz}, 1 \mathrm{H}), 7.89(\mathrm{~d}$, $J=2.0 \mathrm{~Hz}, 1 \mathrm{H}), 7.84-7.78(\mathrm{~m}, 1 \mathrm{H}), 7.54-7.48(\mathrm{~m}, 3 \mathrm{H}), 7.46-$ $7.38(\mathrm{~m}, 2 \mathrm{H}), 2.63-2.52(\mathrm{~m}, 1 \mathrm{H}), 2.24-2.13(\mathrm{~m}, 1 \mathrm{H}), 1.19(\mathrm{~s}$, $3 \mathrm{H}) .{ }^{13} \mathrm{C}$ NMR (100 MHz, $\left.\mathrm{CDCl}_{3} ; \delta, \mathrm{ppm}\right): 201.1,152.1,142.7$, 139.1, 137.0, 136.3, 132.7, 130.1, 129.4, 129.2, 126.7, 124.4, 118.7, 109.6, 50.2, 40.5 (q, $\left.J=27.8 \mathrm{~Hz}, \mathrm{CH}_{2} \mathrm{CF}_{3}\right), 25.1 .{ }^{19} \mathrm{~F}$ NMR (376 $\left.\mathrm{MHz}, \mathrm{CDCl}_{3} ; \delta, \mathrm{ppm}\right):-61.25$ (s, 3F). IR $\left(\mathrm{KBr}, v, \mathrm{~cm}^{-1}\right): 2,205$, $1,726,1,588,1,457,1,419,1,364,1,264,1,179,1,142,1,068,836$, 703. HRMS (ESI, $m / z$ ): calcd for $\mathrm{C}_{20} \mathrm{H}_{13} \mathrm{ClF}_{3} \mathrm{NONa}[\mathrm{M}+\mathrm{Na}]^{+}$ 398.0530, found 398.0491.

(E)-2-[5-Chloro-2-methyl-3-oxo-2-(2,2,2trifluoroethyl)-2,3-dihydro-1H-inden-1-ylidene]-2-(4fluorophenyl)acetonitrile (3l)

Light yellow solid, $48 \mathrm{mg}$, $61 \%$ yield; $\mathrm{mp} 195.2-197.2{ }^{\circ} \mathrm{C} .{ }^{1} \mathrm{H}$ NMR (400 $\left.\mathrm{MHz}, \mathrm{CDCl}_{3} ; \delta, \mathrm{ppm}\right): 8.88$ (d, $\left.J=8.8 \mathrm{~Hz}, 1 \mathrm{H}\right), 7.89$ (d, $J=2.0 \mathrm{~Hz}, 1 \mathrm{H}), 7.84-7.78(\mathrm{~m}, 1 \mathrm{H}), 7.45-7.38(\mathrm{~m}, 2 \mathrm{H}), 7.25-$ $7.18(\mathrm{~m}, 2 \mathrm{H}), 2.68-2.55(\mathrm{~m}, 1 \mathrm{H}), 2.20-2.08(\mathrm{~m}, 1 \mathrm{H}), 1.20(\mathrm{~s}$, $3 \mathrm{H}) .{ }^{13} \mathrm{C} \mathrm{NMR}\left(100 \mathrm{MHz}, \mathrm{CDCl}_{3} ; \delta, \mathrm{ppm}\right): 200.9,163.4\left(\mathrm{~d},{ }^{1} J\right.$ $=250.2 \mathrm{~Hz}, \mathrm{CF}), 152.8,142.4,139.4,137.0,136.4,131.5\left(\mathrm{~d},{ }^{2} \mathrm{~J}\right.$ $=8.4 \mathrm{~Hz}, \mathrm{CF}), 128.6\left(\mathrm{~d},{ }^{3} \mathrm{~J}=3.6 \mathrm{~Hz}, \mathrm{CF}\right), 126.7,124.5,118.6$, $116.7,116.4,108.5,50.1,40.4\left(\mathrm{q}, J=27.8 \mathrm{~Hz}, \mathrm{CH}_{2} \mathrm{CF}_{3}\right), 25.1 .{ }^{19} \mathrm{~F}$ NMR (376 MHz, $\mathrm{CDCl}_{3} ; \delta$, ppm): -61.27(s, 3F), -109.49 (s, 1F). IR $\left(\mathrm{KBr}, v, \mathrm{~cm}^{-1}\right): 2,209,1,727,1,588,1,507,1,426,1,361,1,263$, $1,139,1,064,835$. HRMS (ESI, $m / z$ ): calcd for $\mathrm{C}_{20} \mathrm{H}_{12} \mathrm{ClF}_{4} \mathrm{NONa}$ $[\mathrm{M}+\mathrm{Na}]^{+} 416.0436$, found 416.0421 .
(E)-2-[5-Chloro-2-methyl-3-oxo-2-(2,2,2trifluoroethyl)-2,3-dihydro-1H-inden-1-ylidene]-2-(ptolyl)acetonitrile ( $3 \mathrm{~m}$, Major)

Light yellow solid, $48 \mathrm{mg}$, $62 \%$ yield; mp $121.7-123.6^{\circ} \mathrm{C} .{ }^{1} \mathrm{H} \mathrm{NMR}$ (400 MHz, $\left.\mathrm{CDCl}_{3} ; \delta, \mathrm{ppm}\right): 8.88(\mathrm{~d}, J=8.8 \mathrm{~Hz}, 1 \mathrm{H}), 7.88(\mathrm{~d}$, $J=2.0 \mathrm{~Hz}, 1 \mathrm{H}), 7.81-7.78(\mathrm{~m}, 1 \mathrm{H}), 7.30(\mathrm{~s}, 4 \mathrm{H}), 2.61-2.52(\mathrm{~m}$, $1 \mathrm{H}), 2.43(\mathrm{~s}, 3 \mathrm{H}), 2.26-2.18(\mathrm{~m}, 1 \mathrm{H}), 1.20(\mathrm{~s}, 3 \mathrm{H}) .{ }^{13} \mathrm{C}$ NMR $(100$ $\left.\mathrm{MHz}, \mathrm{CDCl}_{3} ; \delta, \mathrm{ppm}\right): 201.3,152.0,142.8,140.2,139.0,136.3$, $135.4,130.8,129.9,129.3,126.7,124.3,118.8,109.7,50.2,40.4$ (q, $\left.J=27.6 \mathrm{~Hz}, \mathrm{CH}_{2} \mathrm{CF}_{3}\right), 25.2,21.4 .{ }^{19} \mathrm{~F} \mathrm{NMR}\left(376 \mathrm{MHz}, \mathrm{CDCl}_{3}\right.$; $\delta, \mathrm{ppm}):-61.25$ (s, 3F). IR (KBr, $\left.v, \mathrm{~cm}^{-1}\right): 2,205,1,732,1,589$, $1,508,1,457,1,361,1,263,1,178,1,144,1,070,942$, 833. HRMS (ESI, $m / z$ ): calcd for $\mathrm{C}_{21} \mathrm{H}_{15} \mathrm{ClF}_{3} \mathrm{NONa}[\mathrm{M}+\mathrm{Na}]^{+}$412.0686, found 412.0657 .

\section{(E)-2-[5-Chloro-2-methyl-3-oxo-2-(2,2,2-}

trifluoroethyl)-2,3-dihydro-1 $\mathrm{H}$-inden-1-ylidene]-2-(4ethylphenyl)acetonitrile (3n, Major)

Light yellow solid, $48 \mathrm{mg}$, 59\% yield; mp $117.8-120.4^{\circ} \mathrm{C} .{ }^{1} \mathrm{H}$ NMR (400 MHz, $\left.\mathrm{CDCl}_{3} ; \delta, \mathrm{ppm}\right): 8.88(\mathrm{~d}, J=8.8 \mathrm{~Hz}, 1 \mathrm{H}), 7.88(\mathrm{~d}, J=$ $2.0 \mathrm{~Hz}, 1 \mathrm{H}), 7.82-7.78(\mathrm{~m}, 1 \mathrm{H}), 7.32(\mathrm{~s}, 4 \mathrm{H}), 2.75-2.68(\mathrm{~m}, 2 \mathrm{H})$, 2.61-2.53 (m, 1H), 2.27-2.18 (m, $1 \mathrm{H}), 1.30(\mathrm{t}, J=7.6 \mathrm{~Hz}, 3 \mathrm{H})$, $1.20(\mathrm{~s}, 3 \mathrm{H}) .{ }^{13} \mathrm{C} \mathrm{NMR}\left(100 \mathrm{MHz}, \mathrm{CDCl}_{3} ; \delta, \mathrm{ppm}\right): 201.3,152.0$, $146.5,142.8,139.0,136.9,136.3,129.6,129.3,128.7,126.7,124.3$, $118.9,109.8,50.2,40.4\left(\mathrm{q}, J=27.76 \mathrm{~Hz}, \mathrm{CH}_{2} \mathrm{CF}_{3}\right), 28.7,25.1,15.3$. ${ }^{19} \mathrm{~F} \mathrm{NMR}\left(376 \mathrm{MHz}, \mathrm{CDCl}_{3} ; \delta, \mathrm{ppm}\right):-61.24$ (s, 3F). IR (KBr, $v$, $\left.\mathrm{cm}^{-1}\right): 2,203,1,732,1,587,1,507,1,457,1,362,1,254,1,179,1,145$, $1,070,942,833$. HRMS (ESI, $m / z$ ): calcd for $\mathrm{C}_{22} \mathrm{H}_{17} \mathrm{ClF}_{3} \mathrm{NONa}$ $[\mathrm{M}+\mathrm{Na}]^{+}$426.0843, found 426.0824 .

\section{(E)-2-[5-Chloro-2-methyl-3-oxo-2-(2,2,2-} trifluoroethyl)-2,3-dihydro-1 $H$-inden-1-ylidene]-2-(4methoxyphenyl)acetonitrile (3o, Major)

Light yellow solid, $56 \mathrm{mg}, 69 \%$ yield; $\mathrm{mp} 118.4-120.8^{\circ} \mathrm{C} .{ }^{1} \mathrm{H}$ NMR $\left(400 \mathrm{MHz}, \mathrm{CDCl}_{3} ; \delta, \mathrm{ppm}\right): 8.87(\mathrm{~d}, J=8.8 \mathrm{~Hz}, 1 \mathrm{H}), 7.87(\mathrm{~d}, J=$ $2.0 \mathrm{~Hz}, 1 \mathrm{H}), 7.83-7.79(\mathrm{~m}, 1 \mathrm{H}), 7.36-7.33(\mathrm{~m}, 2 \mathrm{H}), 7.02-6.98(\mathrm{~m}$, $2 \mathrm{H}), 3.87(\mathrm{~s}, 3 \mathrm{H}), 2.62-2.54(\mathrm{~m}, 1 \mathrm{H}), 2.27-2.18(\mathrm{~m}, 1 \mathrm{H}), 1.20(\mathrm{~s}$, 3H). ${ }^{13} \mathrm{C}$ NMR (100 MHz, $\mathrm{CDCl}_{3} ; \delta$, ppm): 201.3, 160.7, 152.1, $142.8,138.9,136.9,136.3,130.8,126.7,125.5,124.3,118.9,114.6$, $109.5,55.4,50.2,40.3\left(\mathrm{q}, J=27.8 \mathrm{~Hz}, \mathrm{CH}_{2} \mathrm{CF}_{3}\right), 25.2 .{ }^{19} \mathrm{~F}$ NMR $\left(376 \mathrm{MHz}, \mathrm{CDCl}_{3} ; \delta, \mathrm{ppm}\right):-61.26$ (s, 3F). IR $\left(\mathrm{KBr}, v, \mathrm{~cm}^{-1}\right)$ : 2,203, 1,732, 1,602, 1,508, 1,457, 1,362, 1,255, 1,177, 1,144, 1,069, 833. HRMS (ESI, $m / z$ ): calcd for $\mathrm{C}_{21} \mathrm{H}_{15} \mathrm{ClF}_{3} \mathrm{NO}_{2} \mathrm{Na}[\mathrm{M}+\mathrm{Na}]^{+}$ 428.0636 , found 428.0623 .

(E)-2-[2,5-Dimethyl-3-oxo-2-(2,2,2-trifluoroethyl)-2,3dihydro- $1 H$-inden-1-ylidene]-2- $(p-$ tolyl)acetonitrile (3p)

Light yellow solid, $48 \mathrm{mg}, 65 \%$ yield; $\mathrm{mp} 174.5-176.6^{\circ} \mathrm{C} .{ }^{1} \mathrm{H}$ NMR $\left(400 \mathrm{MHz}, \mathrm{CDCl}_{3} ; \delta, \mathrm{ppm}\right): 8.83(\mathrm{~d}, J=8.8 \mathrm{~Hz}, 1 \mathrm{H}), 7.41-7.37$ (m, $1 \mathrm{H}), 7.31(\mathrm{~d}, J=2.4 \mathrm{~Hz}, 1 \mathrm{H}), 7.29(\mathrm{~s}, 3 \mathrm{H}), 7.26(\mathrm{~s}, 1 \mathrm{H}), 3.94$ (s, 3H), 2.60-2.50 (m, 1H), $2.42(\mathrm{~s}, 3 \mathrm{H}), 2.27-2.16(\mathrm{~m}, 1 \mathrm{H}), 1.19$ (s, 3H). ${ }^{13} \mathrm{C} \mathrm{NMR}\left(100 \mathrm{MHz}, \mathrm{CDCl}_{3} ; \delta\right.$, ppm): 202.4, 163.0, 152.7, $139.8,138.0,137.7,130.2,129.8,129.6,126.8,125.2,119.5,106.5$, $105.6,56.0,50.3,40.3\left(\mathrm{q}, J=27.6 \mathrm{~Hz}, \mathrm{CH}_{2} \mathrm{CF}_{3}\right), 25.2,21.4 .{ }^{19} \mathrm{~F}$ NMR $\left(376 \mathrm{MHz}, \mathrm{CDCl}_{3} ; \delta, \mathrm{ppm}\right):-61.38$ (s, 3F). IR (KBr, $v$, $\left.\mathrm{cm}^{-1}\right): 2,201,1,725,1,594,1,486,1,362,1,296,1,231,1,146,1,069$, 
832. HRMS (ESI, $m / z$ ): calcd for $\mathrm{C}_{22} \mathrm{H}_{18} \mathrm{~F}_{3} \mathrm{NONa}[\mathrm{M}+\mathrm{Na}]^{+}$ 392.1233, found 392.1257 .

(E)-2-(4-Chlorophenyl)-2-[5-methoxy-2-methyl-3oxo-2-(2,2,2-trifluoroethyl)-2,3-dihydro-1 $H$-inden-1ylidene]acetonitrile $(3 \mathrm{q})$

Light yellow solid, $52 \mathrm{mg}$, 64\% yield; mp $130.5-131.2{ }^{\circ} \mathrm{C} .{ }^{1} \mathrm{H}$ NMR (400 MHz, $\left.\mathrm{CDCl}_{3} ; \delta, \mathrm{ppm}\right): 8.83(\mathrm{~d}, J=8.8 \mathrm{~Hz}, 1 \mathrm{H}), 7.48(\mathrm{~d}, J=$ $8.4 \mathrm{~Hz}, 2 \mathrm{H}), 7.40(\mathrm{~d}, J=6.4 \mathrm{~Hz}, 1 \mathrm{H}), 7.39-7.35(\mathrm{~m}, 2 \mathrm{H}), 7.32(\mathrm{~d}$, $J=2.4 \mathrm{~Hz}, 1 \mathrm{H}), 3.94(\mathrm{~s}, 3 \mathrm{H}), 2.68-2.56(\mathrm{~m}, 1 \mathrm{H}), 2.20-2.08(\mathrm{~m}$, $1 \mathrm{H}), 1.19$ (s, 3H). ${ }^{13} \mathrm{C} \mathrm{NMR}\left(100 \mathrm{MHz}, \mathrm{CDCl}_{3} ; \delta, \mathrm{ppm}\right): 201.9$, $163.3,153.5,137.8,137.5,136.1,131.6,131.2,129.5,126.0(\mathrm{q}, J=$ $\left.160.5 \mathrm{~Hz}, \mathrm{CF}_{3}\right), 119.0,105.8,105.0,56.0,50.3,40.3(\mathrm{q}, J=27.7 \mathrm{~Hz}$, $\mathrm{CH}_{2} \mathrm{CF}_{3}$ ), 25.2. ${ }^{19} \mathrm{~F}$ NMR (376 $\left.\mathrm{MHz}, \mathrm{CDCl}_{3} ; \delta, \mathrm{ppm}\right):-61.38$ (s, $3 F)$. IR ( $\left.\mathrm{KBr}, v, \mathrm{~cm}^{-1}\right): 2,202,1,727,1,595,1,488,1,364,1,295$, $1,143,1,019,845$. HRMS (ESI, $m / z$ ): calcd for $\mathrm{C}_{21} \mathrm{H}_{15} \mathrm{ClF}_{3} \mathrm{NO}_{2} \mathrm{Na}$ $[\mathrm{M}+\mathrm{Na}]^{+} 428.0636$, found 428.0616 .

(E)-2-[6-Chloro-2-methyl-3-oxo-2-(2,2,2trifluoroethyl)-2,3-dihydro- $1 \mathrm{H}$-inden-1-ylidene]2-phenylacetonitrile (3r)

Light yellow solid, $53 \mathrm{mg}, 71 \%$ yield; mp $161.6-163.8^{\circ} \mathrm{C} .{ }^{1} \mathrm{H}$ NMR (400 MHz, $\left.\mathrm{CDCl}_{3} ; \delta, \mathrm{ppm}\right): 8.94(\mathrm{~d}, J=1.2 \mathrm{~Hz}, 1 \mathrm{H}), 7.88(\mathrm{~d}$, $J=8.0 \mathrm{~Hz}, 1 \mathrm{H}), 7.69-7.64(\mathrm{~m}, 1 \mathrm{H}), 7.54-7.50(\mathrm{~m}, 3 \mathrm{H}), 7.45-$ $7.39(\mathrm{~m}, 2 \mathrm{H}), 2.62-2.54(\mathrm{~m}, 1 \mathrm{H}), 2.22-2.13(\mathrm{~m}, 1 \mathrm{H}), 1.19(\mathrm{~s}$, $3 \mathrm{H}) .{ }^{13} \mathrm{C} \mathrm{NMR}\left(100 \mathrm{MHz}, \mathrm{CDCl}_{3} ; \delta, \mathrm{ppm}\right): 201.0,151.9,145.7$, $143.3,133.8,133.0,132.6,130.1,129.4,129.3,125.6,125.5,118.4$, $110.5,50.0,40.4\left(\mathrm{q}, J=27.7 \mathrm{~Hz}, \mathrm{CH}_{2} \mathrm{CF}_{3}\right), 25.2 .{ }^{19} \mathrm{~F} \mathrm{NMR}(376$ $\left.\mathrm{MHz}, \mathrm{CDCl}_{3} ; \delta, \mathrm{ppm}\right):-61.25$ (s, 3F). IR $\left(\mathrm{KBr}, v, \mathrm{~cm}^{-1}\right): 2,202$, $1,724,1,589,1,489,1,361,1,271,1,139,1,072,835,712$. HRMS (ESI, $m / z$ ): calcd for $\mathrm{C}_{20} \mathrm{H}_{13} \mathrm{ClF}_{3} \mathrm{NONa}[\mathrm{M}+\mathrm{Na}]^{+}$398.0530, found 398.0556 .

(E)-2-[6-Chloro-2-methyl-3-oxo-2-(2,2,2trifluoroethyl)-2,3-dihydro-1H-inden-1-ylidene]-2-(ptolyl)acetonitrile (3s, Major)

Light yellow solid, $48 \mathrm{mg}$, $62 \%$ yield; $\mathrm{mp} 121.5-123.1{ }^{\circ} \mathrm{C} .{ }^{1} \mathrm{H}$ NMR (400 MHz, $\left.\mathrm{CDCl}_{3} ; \delta, \mathrm{ppm}\right): 8.92(\mathrm{~s}, 1 \mathrm{H}), 7.86(\mathrm{~d}, J=8.0 \mathrm{~Hz}$, $1 \mathrm{H}), 7.65(\mathrm{~d}, J=8.4 \mathrm{~Hz}, 1 \mathrm{H}), 7.36(\mathrm{~d}, J=7.6 \mathrm{~Hz}, 1 \mathrm{H}), 7.30(\mathrm{~s}$, $3 \mathrm{H}), 2.61-2.52(\mathrm{~m}, 1 \mathrm{H}), 2.43(\mathrm{~s}, 3 \mathrm{H}), 2.26-2.17(\mathrm{~m}, 1 \mathrm{H}), 1.19$ (s, $3 \mathrm{H}) .{ }^{13} \mathrm{C} \mathrm{NMR}\left(100 \mathrm{MHz}, \mathrm{CDCl}_{3} ; \delta, \mathrm{ppm}\right): 201.1,151.7,145.8$, 143.2, 140.3, 133.8, 132.9, 130.8, 129.9, 126.8, 125.6, 118.5, 110.7, $50.1,40.4$ (q, $\left.J=27.7 \mathrm{~Hz}, \mathrm{CH}_{2} \mathrm{CF}_{3}\right), 25.2,21.4 .{ }^{19} \mathrm{~F} \mathrm{NMR}(376$ $\left.\mathrm{MHz}, \mathrm{CDCl}_{3} ; \delta, \mathrm{ppm}\right):-61.24$ (s, 3F). IR $\left(\mathrm{KBr}, v, \mathrm{~cm}^{-1}\right): 2,205$, $1,731,1,588,1,509,1,456,1,362,1,255,1,145,1,072,825$. HRMS (ESI, $m / z$ ): calcd for $\mathrm{C}_{21} \mathrm{H}_{15} \mathrm{ClF}_{3} \mathrm{NONa}[\mathrm{M}+\mathrm{Na}]^{+}$412.0686, found 412.0686 .

(E)-2-[2,6-Dimethyl-3-oxo-2-(2,2,2-trifluoroethyl)-2,3dihydro-1H-inden-1-ylidene]-2-phenylacetonitrile (3t) Light yellow solid, $41 \mathrm{mg}$, 58\% yield; mp $147.9-150.4^{\circ} \mathrm{C} .{ }^{1} \mathrm{H} \mathrm{NMR}$ $\left(400 \mathrm{MHz}, \mathrm{CDCl}_{3} ; \delta, \mathrm{ppm}\right): 8.74(\mathrm{~s}, 1 \mathrm{H}), 7.83(\mathrm{~d}, J=8.0 \mathrm{~Hz}$, $1 \mathrm{H}), 7.53-7.48(\mathrm{~m}, 4 \mathrm{H}), 7.45-7.41(\mathrm{~m}, 2 \mathrm{H}), 2.62-2.54(\mathrm{~m}, 4 \mathrm{H})$, 2.21-2.11 (m, 1H), 1.17 (s, 3H). ${ }^{13} \mathrm{C} \mathrm{NMR} \mathrm{(100} \mathrm{MHz,} \mathrm{CDCl}_{3}$; $\delta$, ppm): 201.7, 153.3, 147.9, 144.8, 133.7, 133.5, 133.1, 129.8, $129.6,129.2,125.5\left(\mathrm{q}, J=112.6 \mathrm{~Hz}, \mathrm{CF}_{3}\right), 119.0,108.7,50.0$, $40.3\left(\mathrm{q}, J=27.6 \mathrm{~Hz}, \mathrm{CH}_{2} \mathrm{CF}_{3}\right), 25.2,22.6 .{ }^{19} \mathrm{~F} \mathrm{NMR}(376 \mathrm{MHz}$,
$\left.\mathrm{CDCl}_{3} ; \delta, \mathrm{ppm}\right):-61.33$ (s, 3F). IR (KBr, $\left.v, \mathrm{~cm}^{-1}\right): 2,202,1,716$, $1,613,1,489,1,455,1,360,1,253,1,136,1,072,831,767$. HRMS (ESI, $m / z$ ): calcd for $\mathrm{C}_{21} \mathrm{H}_{16} \mathrm{~F}_{3} \mathrm{NONa}[\mathrm{M}+\mathrm{Na}]^{+}$378.1076, found 378.1054 .

(E)-2-(4-Chlorophenyl)-2-[2,6-dimethyl-3-oxo-2(2,2,2-trifluoroethyl)-2,3-dihydro- $1 \mathrm{H}$-inden-1ylidene]acetonitrile

\section{(3u, Major)}

Light yellow solid, $66 \mathrm{mg}$, 85\% yield; mp $108.1-110.8^{\circ} \mathrm{C} .{ }^{1} \mathrm{H} \mathrm{NMR}$ $\left(400 \mathrm{MHz}, \mathrm{CDCl}_{3} ; \delta, \mathrm{ppm}\right): 8.72(\mathrm{~s}, 1 \mathrm{H}), 7.84(\mathrm{~d}, J=8.0 \mathrm{~Hz}, 1 \mathrm{H})$, $7.52-7.48(\mathrm{~m}, 3 \mathrm{H}), 7.40(\mathrm{~d}, J=6.8 \mathrm{~Hz}, 2 \mathrm{H}), 2.66-2.58(\mathrm{~m}, 4 \mathrm{H})$, 2.17-2.08 (m, 1H), $1.18(\mathrm{~s}, 3 \mathrm{H}) .{ }^{13} \mathrm{C} \mathrm{NMR}\left(100 \mathrm{MHz}, \mathrm{CDCl}_{3} ; \delta\right.$, ppm): 201.3, 154.0, 148.0, 144.6, 136.2, 134.0, 133.8, 132.3, 131.0, $129.5,126.9,125.0\left(\mathrm{q}, J=106.5 \mathrm{~Hz}, \mathrm{CF}_{3}\right), 124.6,118.6,107.4$, 50.0, $40.4\left(\mathrm{q}, J=27.6 \mathrm{~Hz}, \mathrm{CH}_{2} \mathrm{CF}_{3}\right), 25.2,22.6 .{ }^{19} \mathrm{~F}$ NMR $(376$ $\left.\mathrm{MHz}, \mathrm{CDCl}_{3} ; \delta, \mathrm{ppm}\right):-61.33$ (s, 3F). IR $\left(\mathrm{KBr}, v, \mathrm{~cm}^{-1}\right): 2,208$, $1,727,1,595,1,489,1,360,1,253,1,180,1,142,1,071,832$. HRMS (ESI, $m / z$ ): calcd for $\mathrm{C}_{21} \mathrm{H}_{15} \mathrm{ClF}_{3} \mathrm{NONa}[\mathrm{M}+\mathrm{Na}]^{+}$412.0686, found 412.0637 .

\section{(E)-2-(4-Bromophenyl)-2-[2,6-dimethyl-3-oxo-2-} (2,2,2-trifluoroethyl)-2,3-dihydro- $1 H$-inden-1ylidene]acetonitrile

\section{(3v, Major)}

Light yellow solid, $39 \mathrm{mg}, 45 \%$ yield; $\mathrm{mp} 136.5-138.6^{\circ} \mathrm{C} .{ }^{1} \mathrm{H}$ NMR $\left(400 \mathrm{MHz}, \mathrm{CDCl}_{3} ; \delta, \mathrm{ppm}\right): 8.71(\mathrm{~s}, 1 \mathrm{H}), 7.83(\mathrm{~d}, J=8.0 \mathrm{~Hz}, 1 \mathrm{H})$, $7.65(\mathrm{~d}, J=8.4 \mathrm{~Hz}, 2 \mathrm{H}), 7.52(\mathrm{~d}, J=8.0 \mathrm{~Hz}, 1 \mathrm{H}), 7.31(\mathrm{~d}, J=$ $8.4 \mathrm{~Hz}, 2 \mathrm{H}), 2.66-2.61(\mathrm{~m}, 1 \mathrm{H}), 2.58(\mathrm{~s}, 3 \mathrm{H}), 2.17-2.09(\mathrm{~m}, 1 \mathrm{H})$, 1.18 (s, 3H). ${ }^{13} \mathrm{C}$ NMR (100 MHz, $\mathrm{CDCl}_{3} ; \delta$, ppm): 201.3, 154.0, $145.0,144.6,134.0,133.8,133.2,132.5,131.2,126.9,125.5(\mathrm{q}, J$ $\left.=105.5 \mathrm{~Hz}, \mathrm{CF}_{3}\right), 124.6,118.5,107.4,50.0,40.4(\mathrm{q}, J=27.7 \mathrm{~Hz}$, $\left.\mathrm{CH}_{2} \mathrm{CF}_{3}\right), 25.2,22.6 .{ }^{19} \mathrm{~F}$ NMR $\left(376 \mathrm{MHz}, \mathrm{CDCl}_{3} ; \delta, \mathrm{ppm}\right)$ : -61.31 (s, 3F). IR ( $\left.\mathrm{KBr}, v, \mathrm{~cm}^{-1}\right): 2,206,1,731,1,593,1,456$, $1,362,1,255,1,141,1,070,1,011,831$. HRMS (ESI, $m / z)$ : calcd for $\mathrm{C}_{21} \mathrm{H}_{15} \mathrm{BrF}_{3} \mathrm{NONa}[\mathrm{M}+\mathrm{Na}]^{+}$456.0181, found 456.0137.

(E)-2-[2,6-Dimethyl-3-oxo-2-(2,2,2-trifluoroethyl)-2,3dihydro- $1 H$-inden-1-ylidene]-2-( $p$ tolyl)acetonitrile (3w, Major)

Light yellow solid, $54 \mathrm{mg}$, $73 \%$ yield; $\mathrm{mp} 127.8-129.9^{\circ} \mathrm{C} .{ }^{1} \mathrm{H}$ NMR $\left(400 \mathrm{MHz}, \mathrm{CDCl}_{3} ; \delta, \mathrm{ppm}\right): 8.73(\mathrm{~s}, 1 \mathrm{H}), 7.82(\mathrm{~d}, J=8.0 \mathrm{~Hz}, 1 \mathrm{H})$, 7.49 (d, $J=8.0 \mathrm{~Hz}, 1 \mathrm{H}), 7.30(\mathrm{~s}, 4 \mathrm{H}), 2.61-2.53(\mathrm{~m}, 4 \mathrm{H}), 2.43$ (s, 3H), 2.24-2.17 (m, 1H), 1.18 (s, 3H). ${ }^{13} \mathrm{C}$ NMR (100 MHz, $\left.\mathrm{CDCl}_{3} ; \delta, \mathrm{ppm}\right): 201.9,153.2,147.8,144.9,140.0,133.6,133.3$, $130.5,129.8,129.4,127.1,125.4\left(\mathrm{q}, J=114.4 \mathrm{~Hz}, \mathrm{CF}_{3}\right), 119.1$, $108.9,50.0,40.3\left(\mathrm{q}, J=27.5 \mathrm{~Hz}, \mathrm{CH}_{2} \mathrm{CF}_{3}\right), 25.2,22.6,21.4 .{ }^{19} \mathrm{~F}$ NMR $\left(376 \mathrm{MHz}, \mathrm{CDCl}_{3} ; \delta, \mathrm{ppm}\right):-61.32(\mathrm{~s}, 3 \mathrm{~F})$. IR ( $\mathrm{KBr}, v$, $\left.\mathrm{cm}^{-1}\right): 2,204,1,719,1,609,1,590,1,510,1,456,1,361,1,254,1,144$, $1,071,830$. HRMS (ESI, $m / z$ ): calcd for $\mathrm{C}_{22} \mathrm{H}_{18} \mathrm{~F}_{3} \mathrm{NONa}[\mathrm{M}+$ $\mathrm{Na}]^{+}$392.1233, found 392.1223.

(E)-2-[2,6-Dimethyl-3-oxo-2-(2,2,2-trifluoroethyl)-2,3dihydro-1 $H$-inden-1-ylidene]-2-(4-methoxyphenyl) acetonitrile (3x, Major)

Light yellow solid, $63 \mathrm{mg}, 82 \%$ yield; $\mathrm{mp} 120.8-122.4^{\circ} \mathrm{C} .{ }^{1} \mathrm{H}$ NMR (400 MHz, $\left.\mathrm{CDCl}_{3} ; \delta, \mathrm{ppm}\right): 8.72(\mathrm{~s}, 1 \mathrm{H}), 7.82(\mathrm{~d}, J=8.0 \mathrm{~Hz}$, 
$1 \mathrm{H}), 7.49(\mathrm{~d}, J=8.0 \mathrm{~Hz}, 1 \mathrm{H}), 7.36(\mathrm{~d}, J=8.4 \mathrm{~Hz}, 2 \mathrm{H}), 7.00$ (d, $J=8.8 \mathrm{~Hz}, 2 \mathrm{H}), 3.87$ (s, 3H), 2.62-2.55 (m, 4H), 2.24-2.19 $(\mathrm{m}, 1 \mathrm{H}), 1.19(\mathrm{~s}, 3 \mathrm{H}) .{ }^{13} \mathrm{C} \mathrm{NMR}\left(100 \mathrm{MHz}, \mathrm{CDCl}_{3} ; \delta, \mathrm{ppm}\right)$ : $201.9,160.5,153.3,147.8,144.9,133.6,133.3,130.9,127.0,125.5$ $\left(\mathrm{q}, J=114.2 \mathrm{~Hz}, \mathrm{CF}_{3}\right), 125.1,119.2,114.5,108.6,55.4,50.1$, $40.2\left(\mathrm{q}, J=27.7 \mathrm{~Hz}, \mathrm{CH}_{2} \mathrm{CF}_{3}\right), 25.2,22.6 .{ }^{19} \mathrm{~F} \mathrm{NMR}(376 \mathrm{MHz}$, $\left.\mathrm{CDCl}_{3} ; \delta, \mathrm{ppm}\right):-61.32(\mathrm{~s}, 3 \mathrm{~F})$. IR $\left(\mathrm{KBr}, v, \mathrm{~cm}^{-1}\right): 2,205,1,724$, $1,605,1,507,1,457,1,362,1,257,1,141,1,070,1,026,832$. HRMS (ESI, $m / z$ ): calcd for $\mathrm{C}_{22} \mathrm{H}_{18} \mathrm{~F}_{3} \mathrm{NO}_{2} \mathrm{Na}[\mathrm{M}+\mathrm{Na}]^{+}$408.1182, found 408.1182 .

\section{3-[2-Amino-1-(4-chlorophenyl)ethyl]-6-methoxy-2- methyl-2-(2,2,2-trifluoroethyl)-2,3-dihydro-1H-inden- 1-ol (4)}

White oil, $11 \mathrm{mg}, 51 \%$ yield. ${ }^{1} \mathrm{H}$ NMR (400 $\mathrm{MHz}, \mathrm{CDCl}_{3}$; $\delta, \mathrm{ppm}): 8.39(\mathrm{~d}, J=8.4 \mathrm{~Hz}, 1 \mathrm{H}), 7.47-7.41(\mathrm{~m}, 2 \mathrm{H}), 7.35-$ $7.30(\mathrm{~m}, 2 \mathrm{H}), 6.99(\mathrm{~s}, 2 \mathrm{H}), 4.78(\mathrm{~s}, 1 \mathrm{H}), 3.89(\mathrm{~s}, 3 \mathrm{H}), 3.70-$ $3.66(\mathrm{~m}, 1 \mathrm{H}), 3.25-3.21(\mathrm{~m}, 1 \mathrm{H}), 2.37-2.23(\mathrm{~m}, 2 \mathrm{H}), 1.95-$ $1.91(\mathrm{~m}, 1 \mathrm{H}), 1.78(\mathrm{~s}, 2 \mathrm{H}), 1.70-1.66(\mathrm{~m}, 1 \mathrm{H}), 1.07(\mathrm{~s}, 3 \mathrm{H})$. ${ }^{13} \mathrm{C}$ NMR (100 MHz, $d_{6}$-DMSO; $\left.\delta, \mathrm{ppm}\right): 162.8,160.4,151.7$, $134.3,133.5,130.7$ (q, $\left.J=154.3 \mathrm{~Hz}, \mathrm{CF}_{3}\right), 128.2,126.1,120.2$, $115.7,109.0,102.2,79.4,67.5,56.0,53.7,25.6,24.2 .{ }^{19} \mathrm{~F}$ NMR (376 MHz, $d_{6}$-DMSO; $\left.\delta, \mathrm{ppm}\right):-58.24$ (s, 3F). HRMS (ESI,

\section{REFERENCES}

Bergmann, E. D. (1956). Fulvenes and thermochromic ethylenes. Part 30. experiments in the 2,3-diarylindone series. J. Org. Chem. 21, 461-464. doi: 10.1021/jo01110a023

Chen, M., Weng, Y., Guo, M., Zhang, H., and Lei, A. (2008). Nickel-catalyzed reductive cyclization of unactivated 1,6-enynes in the presence of organozinc reagents. Angew. Chem. Int. Ed. 47, 2279-2282. doi: 10.1002/anie.200704452

Chen, S., Feng, D.-F., Li, D.-Y., and Liu, P.-N. (2018). Radical cyanotrifluoromethylation of isocyanides: step-economical access to $\mathrm{CF}_{3}$-containing nitriles, amines, and imines. Org. Lett. 20, 5418-5422. doi: 10.1021/acs.orglett.8b02328

Chen, Z.-M., Bai, W., Wang, S.-H., Yan, B.-M., Tu, Y.-Q., and Zhang, F.-M. (2013). Copper-catalyzed tandem trifluoromethylation/semipinacol rearrangement of allylic alcohols. Angew. Chem. Int. Ed. 52, 9781-9785. doi: 10.1002/anie.201304557

Chernyak, N., Gorelsky, S. I., and Gevorgyan, V. (2011). Palladium-catalyzed carbocyclization of alkynyl ketones proceeding through a carbopalladation pathway. Angew. Chem. Int. Ed. 50, 2342-2345. doi: 10.1002/anie.201006751

Chu, L., and Qing, F.-L. (2012). Copper-catalyzed oxidative trifluoromethylation of terminal alkenes using nucleophilic $\mathrm{CF}_{3} \mathrm{SiMe}_{3}$ : efficient $\mathrm{C}\left(\mathrm{sp}^{3}\right)-\mathrm{CF}^{3}$ bond formation. Org. Lett. 14, 2106-2109. doi: 10.1021/ol300639a

Dai, J.-R., Hallock, Y. F., Cardellina, J. H., and Boyd, M. R. (1998). HIV-inhibitory and cytotoxic oligostilbenes from the leaves of Hopea malibato. J. Nat. Prod.61, 351-353. doi: 10.1021/np970519h

deSolms S. J., Woltersdorf O. W. Jr., Cragoe E. J. Jr., Watson L. S., and Fanelli G. M. Jr. (1978). (Acylaryloxy)acetic acid diuretics. 2. (2-Alkyl-2-Aryl-1-Oxo-5Indanyloxy)acetic acids. J. Med. Chem. 21, 437-443. doi: 10.1021/jm00203a006

Egami, H., Shimizu, R., and Sodeoka, M. (2012). Oxytrifluoromethylation of multiple bonds using copper catalyst under mild conditions. Tetrahedron Lett. 53, 5503-5506. doi: 10.1016/j.tetlet.2012.07.134

Egami, H., Shimizu, R., Usui, Y., and Sodeoka, M. (2013). Iron-catalyzed trifluoromethylation with concomitant $\mathrm{C}$-C bond formation via 1,2-migration of an aryl group. Chem. Commun. 49, 7346-7348. doi: 10.1039/c3cc43936d

Frank, R. L., Eklund, H., Richter, J. W., Vanneman, C. R., and Wennerberg, A. N. (1944). Some 2,3-disubstituted indones. J. Am. Chem. Soc. 66, 1-4. doi: $10.1021 /$ ja01229a001 $m / z$ ): calcd for $\mathrm{C}_{21} \mathrm{H}_{23} \mathrm{ClF}_{3} \mathrm{NNaO}_{2}[\mathrm{M}+\mathrm{Na}]^{+}$436.8508, found 436.8517 .

\section{DATA AVAILABILITY STATEMENT}

The datasets generated for this study are available on request to the corresponding author.

\section{AUTHOR CONTRIBUTIONS}

T-SZ, BJ, and P-JC designed the project. T-SZ performed the experiments. T-SZ, W-JH, S-JT, and P-JC analyzed the data. T-SZ, $\mathrm{BJ}$, and GL wrote the manuscript.

\section{ACKNOWLEDGMENTS}

We are grateful for financial support from the Fundamental Research Funds for the Central Universities (JH180263).

\section{SUPPLEMENTARY MATERIAL}

The Supplementary Material for this article can be found online at: https://www.frontiersin.org/articles/10.3389/fchem. 2020.00234/full\#supplementary-material

Fu, W., Guo, W., Zou, G., and Xu, C. (2012). Selective trifluoromethylation and alkynylation of tetrahydroisoquinolines using visible light irradiation by Rose Bengal. J. Fluor. Chem. 140, 88-94. doi: 10.1016/j.jfluchem.2012. 05.009

Ge, G.-C., Huang, X.-J., Ding, C.-H., Wan, S.-L., Dai, L.-X., and Hou, X.-L. (2014). A new strategy to construct a $\mathrm{C}-\mathrm{C}-\mathrm{CF}_{3}$ subunit via $\mathrm{CuBr}$ catalyzed domino reaction of homopropargyl amines: an efficient synthesis of trifluoromethyl containing building blocks 4-trifluoromethyl-2,3dihydro-pyrroliums. Chem. Commun. 50, 3048-3051. doi: 10.1039/c3cc4 9059a

Hagmann, W. K. (2008). The many roles for fluorine in medicinal chemistry. $J$. Med. Chem. 51, 4359-4369. doi: 10.1021/jm800219f

Hao, W.-J., Gao, Q., Jiang, B., Liu, F., Wang, S.-L., Tu, S.-J., et al. (2016). Base-promoted $[4+1] /[3+1+1]$ bicyclization for accessing functionalized Indeno[1,2-c]furans. J. Org. Chem. 81, 11276-11281. doi: 10.1021/acs.joc.6b02249

He, G., Wu, C., Zhou, J., Yang, Q., Zhang, C., Zhou, Y., et al. (2018). A method for synthesis of 3-Hydroxy-1-indanones via Cu-catalyzed intramolecular annulation reactions. J. Org. Chem. 83, 13356-13362. doi: 10.1021/acs.joc.8b02149

He, Y.-T., Li, L.-H., Yang, Y.-F., Zhou, Z.-Z., Hua, H.-L., Liu, X.-, and Liang, Y.-M. (2014a). Copper-catalyzed intermolecular cyanotrifluoromethylation of alkenes. Org. Lett. 16, 270-273. doi: 10.1021/ol403263c

He, Y.-T., Li, L.-H., Zhou, Z.-Z., Hua, H.-L., Qiu, Y.-F., Liu, X.-Y., et al. (2014b). Copper-catalyzed three-component cyanotrifluoromethylation/azidotrifluoromethylation and carbocyclization of 1,6-enynes. Org. Lett. 16, 3896-3899. doi: 10.1021/ol501574f

He, Y.-T., Wang, Q., Zhao, J., Liu, X.-Y., Xu, P.-F., and Liang, Y.-M. (2015). The copper-catalyzed synthesis of $\beta$-trifluoromethylated acrylonitriles and trifluoromethyl-substituted 2H-azirines. Chem. Commun. 51, 13209-13212. doi: 10.1039/C5CC05066A

Huang, M.-H., Hao, W.-J., Li, G., Tu, S.-J., and Jiang, B. (2018). Recent advances in radical transformations of internal alkynes. Chem. Commun. 54, 10791-10811. doi: 10.1039/C8CC04618B

Iqbal, N., Jung, J., Park, S., and Cho, E. J. (2014). Controlled trifluoromethylation reactions of alkynes through visible-light photoredox catalysis. Angew. Chem. Int. Ed. 53, 539-542. doi: 10.1002/anie.201308735 
Ito, T., Tanaka, T., Iinuma, M., Nakaya, K., Takahashi, Y., Sawa, R., et al. (2004). Three new resveratrol oligomers from the stem bark of Vatica pauciflora. J. Nat. Prod. 67, 932-937. doi: 10.1021/np030236r

Janson, P. G., Ghoneim, I., Ilchenko, N. O., and Szabó, K. J. (2012). Electrophilic trifluoromethylation by copper-catalyzed addition of $\mathrm{CF}_{3}$-transfer reagents to Alkenes and Alkynes. Org. Lett. 14, 2882-2885. doi: 10.1021/ol3011419

Ji, C.-L., Hao, W.-J., Zhang, J., Geng, F.-Z., Xu, T., Tu, S.-J., et al. (2019). Catalytic three-component synthesis of functionalized naphtho[2,1-b] oxecines via a double bond cleavage-rearrangement cascade. Org. Lett. 21, 6494-6498. doi: 10.1021 /acs.orglett.9b02367

Kamigata, N., Fukushima, T., and Yoshida, M. (1990). Reactions of perfluoroalkanesulfonyl chlorides with aromatic compounds catalyzed by a Ruthenium(II) complex. Chem. Lett. 649-650. doi: 10.1246/cl.1990.649

Koelsch, C. F. (1932). Synthesis with triarylvinylmagnesium romides. triarylacrylic acids and the indones derived from them. J. Am. Chem. Soc. 54, 2487-2493. doi: 10.1021/ja01345a046

Kong, W., Fuentes, N., García-Domínguez, A., Merino, E., and Nevado, C. (2015). Stereoselective synthesis of highly functionalized indanes and dibenzocycloheptadienes through complex radical cascade reactions. Angew. Chem. Int. Ed. 54, 2487-2491. doi: 10.1002/anie.201409659

Kuninobu, Y., Nagase, M., and Kanai, M. (2015). Benzylic C(sp $\left.{ }^{3}\right)-\mathrm{H}$ perfluoroalkylation of six-membered heteroaromatic compounds. Angew. Chem. Int. Ed. 54, 10263-10266. doi: 10.1002/anie.201505335

Li, Y., and Studer, A. (2012). Transition-metal-free trifluoromethylaminoxylation of alkenes. Angew. Chem. Int. Ed. 51, 8221-8224. doi: 10.1002/anie.201202623

Lin, J.-S., Dong, X.-Y., Li, T.-T., Jiang, N.-C., Tan, B., and Liu, X.-Y. (2016). A dual-catalytic strategy to direct asymmetric radical aminotrifluoromethylation of alkenes. J. Am. Chem. Soc. 138, 9357-9360. doi: 10.1021/jacs.6b04077

Liu, S., Chen, K., Hao, W.-J., Tu, X.-C., Tu, S.-J., and Jiang, B. (2019). Generation of 1,3-dimethylene-substituted isobenzofurans via $\mathrm{Pd}(\mathrm{II})$-catalyzed selective oxo-cyclization $/ \mathrm{SO}_{2}$ insertion cascade of $\beta$-Alkynyl ketones. J. Org. Chem. 84, 1964-1971. doi: 10.1021/acs.joc.8b02934

Liu, W., Huang, X., Cheng, M.-J., Nielsen, R. J., Goddard, III W. A., and Groves, J. T. (2012). Oxidative aliphatic C-H fluorination with fluoride ion catalyzed by a manganese porphyrin. Science. 337, 1322-1325. doi: 10.1126/science.1222327

Liu, X., Xiong, F., Huang, X., Xu, L., Li, P., and Wu, X. (2013). Copper-Catalyzed trifluoromethylation-initiated radical 1,2-Aryl Migration in $\alpha, \alpha$-diaryl allylic alcohols. Angew. Chem. Int. Ed. 52, 6962-6966. doi: 10.1002/anie.201302673

Liu, Y., Zhang, J.-L., Song, R.-J., Qian, P.-C., and Li, J.-H. (2014). Cascade Nitration/Cyclization of 1,7-Enynes with ${ }^{t} \mathrm{BuONO}$ and $\mathrm{H}_{2} \mathrm{O}$ : one-pot self-assembly of Pyrrolo[4,3,2-de]quinolinones. Angew. Chem. Int. Ed. 53, 9017-9020. doi: 10.1002/anie.201404192

Manning, C., McClory, M. R., and McCullough, J. J. (1981). Sigmatropic Rearrangements of 1,1-diarylindenes. Migratory aptitudes of aryl migration in the ground and electronically excited states. J. Org. Chem. 46, 919-930. doi: 10.1021/jo00318a018

Müller, K., Faeh, C., and Diederich, F. (2007). Fluorine in pharmaceuticals: looking beyond intuition. Science 317, 1881-1886. doi: 10.1126/science.1131943

Nagle, D. G., Zhou, Y.-D., Park, P. U., Paul, V. J., Rajbhandari, I., Duncan, C. J. G., et al. (2000). A new indanone from the marine cyanobacterium lyngbya majuscula that inhibits hypoxia-induced activation of the VEGF promoter in $\mathrm{Hep}_{3}$ B cells. J. Nat. Prod. 63, 1431-1433. doi: 10.1021/np 000216e

Nitta, T., Arai, T., Takamatsu, H., Inatomi, Y., Murata, H., Iinuma, M., et al. (2002). Antibacterial activity of extracts prepared from tropical and subtropical plants on methicillin-resistant Staphylococcus aureus. J. Health Sci. 48, 273-276. doi: $10.1248 /$ hhs. 48.273

Pair, E., Monteiro, N., Bouyssi, D., and Baudoin, O. (2013). Copper-catalyzed trifluoromethylation of $\mathrm{N}, \mathrm{N}$-dialkylhydrazones. Angew. Chem. Int. Ed. 52, 5346-5349. doi: 10.1002/anie.201300782

Pan, Y., Wang, S., Kee, C. W., Dubuisson, E., Yang, Y., Loh, K. P., et al. (2011). Graphene oxide and Rose Bengal: oxidative C-H functionalisation of tertiary amines using visible light. Green Chem. 13, 3341-3344. doi: $10.1039 / \mathrm{clgc} 15865 \mathrm{a}$

Qin, X.-Y., He, L., Li, J., Hao, W.-J., Tu, S.-J., and Jiang, B. (2019). Regioselective synthesis of polycyclic sulfones via radical-induced threecomponent bicyclization cascades. Chem. Commun. 55, 3227-3230. doi: 10.1039/C9CC00324J
Shen, Z.-J., Shi, H.-N., Hao, W.-J., Tu, S.-J., and Jiang, B. (2018b). Visiblelight photocatalytic bicyclization of $\beta$-alkynyl propenones for accessing diastereoenriched syn-fluoren-9-ones. Chem. Commun. 54, 11542-11545. doi: 10.1039/C8CC06086J

Shen, Z.-J., Wang, S.-C., Hao, W.-J., Yang, S.-Z., Tu, S.-J., and Jiang, B. (2019). Switching between copper-catalysis and photocatalysis for tunable halofluoroalkylation and hydrofluoroalkylation of 1,6-enynes toward 1indenones. Adv. Synth. Catal. 361, 3837-3851. doi: 10.1002/adsc.201900559

Shen, Z.-J., Wu, Y.-N., He, C.-L., He, L., Hao, W.-J., Wang, A.-F., et al. (2018a). Stereoselective synthesis of sulfonated 1-indenones via radical-triggered multicomponent cyclization of $\beta$-alkynyl propenones. Chem. Commun. 54, 445-448. doi: 10.1039/C7CC08516H

Shi, H.-N., Huang, M.-H., Hao, W.-J., Tu, X.-C., Tu, S.-J., and Jiang, B. (2019a). Synthesis of diastereoenriched 1-indanones via double-Base cooperatively Promoted 1,4-Oxo-Migration/Cyclization of $\beta$-Alkynyl Ketones. J. Org. Chem. 84, 16027-16035. doi: 10.1021/acs.joc.9b02525

Shi, H.-N., Huang, M.-H., He, C.-L., Lu, H.-P., Hao, W.-J., Tu, X.-C., et al. (2019b). Completely stereoselective synthesis of sulfonated 1,3-dihydroisobenzofurans via radical multicomponent reactions. J. Org. Chem. 84, 13686-13695. doi: 10.1021 /acs.joc.9b01918

Shimizu, R., Egami, H., Hamashima, Y., and Sodeoka, M. (2012). Copper-catalyzed trifluoromethylation of allylsilanes. Angew. Chem., Int. Ed. 51, 4577-4480. doi: 10.1002/anie. 201201095

Shintani, R., Takatsu, K., and Hayashi, T. (2007). Rhodium-catalyzed asymmetric synthesis of 3,3-disubstituted 1-indanones. Angew. Chem. Int. Ed. 46, 3735-3737. doi: 10.1002/anie.200700226

Song, L., Tian, G., and Van der Eycken, E. V. (2019). Chemo- and regioselective catalyst-controlled carbocyclization of alkynyl ketones: rapid synthesis of 1-indanones and 1-naphthols. Chem. Eur. J. 25, 7645-7648. doi: $10.1002 /$ chem. 201901860

Studer, A. (2012). A "Renaissance" in radical trifluoromethylation. Angew. Chem. Int. Ed. 51, 8950-8958. doi: 10.1002/anie.201202624

Tomita, R., Koike, T., and Akita, M. (2015). Photoredox-catalyzed stereoselective conversion of alkynes into tetrasubstituted trifluoromethylated alkenes. Angew. Chem. Int. Ed. 54, 12923-12927. doi: 10.1002/anie.201505550

Umemoto, T. (1996). Electrophilic perfluoroalkylating agents. Chem. Rev. 96, 1757-1778. doi: $10.1021 / \mathrm{cr} 941149 \mathrm{u}$

Wang, F., Wang, D., Wan, X., Wu, L., Chen, P., and Liu, G. (2016). Enantioselective copper-catalyzed intermolecular cyanotrifluoromethylation of alkenes via radical process. J. Am. Chem. Soc. 138, 15547-15550. doi: 10.1021/jacs.6b10468

Wang, F., Zhu, N., Chen, P., Ye, J., and Liu, G. (2015). Copper-catalyzed trifluoromethylazidation of alkynes: efficient access to $\mathrm{CF}_{3}$-substituted azirines and aziridines. Angew. Chem. Int. Ed. 54, 9356-9360. doi: $10.1002 /$ anie. 201503412

Wang, N.-N., Hao, W.-J., Zhang, T.-S., Li, G., Wu, Y.-N., Tu, S.-J., et al. (2016a). Metal-free $\mathrm{C}\left(\mathrm{sp}^{3}\right)$-H functionalization: oxidative carbo-oxygenation of $\alpha$-diazo carbonyls via radical dediazotization. Chem. Commun. 52, 5144-5147. doi: 10.1039/C6CC00816J

Wang, N.-N., Huang, L.-R., Hao, W.-J., Zhang, T.-S., Li, G., Tu, S.-J., et al. (2016b). Synergistic rhodium/copper catalysis: synthesis of 1,3-enynes and $N$-Aryl enaminones. Org. Lett. 18, 1298-1301. doi: 10.1021/acs.orglett.6b00238

Wessig, P., and Teubner, J. (2006). Total synthesis of pterosines B and C via a photochemical key step. Synlett 2006, 1543-1546. doi: 10.1055/s-2006-944190

Wilger, D. J., Gesmundo, N. J., and Nicewicz, D. A. (2013). Catalytic hydrotrifluoromethylation of styrenes and unactivated aliphatic alkenesvia an organic photoredox system. Chem. Sci. 4, 3160-3165. doi: 10.1039/c3sc5 $1209 \mathrm{f}$

Wu, X., Chu, L., and Qing, F.-L. (2013). Silver-catalyzed hydrotrifluoromethylation of unactivated alkenes with $\mathrm{CF}_{3} \mathrm{SiMe}_{3}$. Angew. Chem. Int. Ed. 52, 2198-2202. doi: 10.1002/anie.201208971

Wu, Z., Wang, D., Liu, Y., Huan, L., and Zhu, C. (2017). Chemoand regioselective distal heteroaryl ipso-Migration: a general protocol for heteroarylation of unactivated alkenes. J. Am. Chem. Soc. 139, 1388-1391. doi: 10.1021/jacs.6b11234

Xiao, H., Liu, Z., Shen, H., Zhang, B., Zhu, L., and Li, C. (2019). Copper-catalyzed late-stage benzylic $\mathrm{C}\left(\mathrm{sp}^{3}\right)-\mathrm{H}$ trifluoromethylation. Chemistry 5, 940-949. doi: 10.1016/j.chempr.2019. 02.006 
Yang, X., Wu, T., Phipps, R. J., and Toste, F. D. (2015). Advances in catalytic enantioselective fluorination, Mono-, Di-, and trifluoromethylation, and trifluoromethylthiolation reactions. Chem. Rev. 115, 826-870. doi: $10.1021 /$ cr500277b

Yasu, Y., Koike, T., and Akita, M. (2013). Visible-light-induced synthesis of a variety of trifluoromethylated alkenes from potassium vinyltrifluoroborates by photoredox catalysis. Chem. Commun. 49, 2037-2039. doi: 10.1039/c3cc39235j

Zhang, Z. Q., Meng, X. Y., Sheng, J., Lan, Q., and Wang, X. S. (2019). Enantioselective copper-catalyzed 1, 5cyanotrifluoromethylation of vinylcyclopropanes. Organic Lett. 21, 8256-8260. doi: 10.1021/acs.orglett.9b03012

Zhu, R., and Buchwald, S. L. (2012). Copper-catalyzed oxytrifluoromethylation of unactivated alkenes. J. Am. Chem. Soc. 134, 12462-12465. doi: 10.1021/ja $305840 \mathrm{~g}$

Zhu, R., and Buchwald, S. L. (2013). Enantioselective functionalization of radical intermediates in redox catalysis: copper-catalyzed asymmetric oxytrifluoromethylation of alkenes. Angew. Chem. Int. Ed. 52, 12655-12658. doi: 10.1002/anie.20130 7790

Zhu, X.-T., Lu, Q.-L., Wang, X., Zhang, T.-S., Hao, W.-J., Tu, S.-J., et al. (2018a). Substrate-controlled generation of 3-sulfonylated 1indenones and 3 -arylated $(Z)$-indenes via $\mathrm{Cu}$-catalyzed radical cyclization cascades of o-Alkynylbenzonitriles. J. Org. Chem. 83, 9890-9901. doi: 10.1021/acs.joc.8b01343

Zhu, X.-T., Zhang, T.-S., Zhao, Q., Cai, P.-J., Hao, W.-J., Tu, S.-J., et al. (2018b). Sulfinate-salt-mediated radical relay cyclization of cyclic ethers with 2-alkynylbenzonitriles toward 3-alkylated 1-indenones. Chem. Asian J. 13, 1157-1164. doi: 10.1002/asia.201800211

Zhu, X.-T., Zhao, Q., Liu, F., Wang, A.-F., Cai, P.-J., Hao, W.-J., et al. (2017). Silver-mediated radical 5-exo-dig cyclization of 2-alkynylbenzonitriles: synthesis of phosphinylated 1-indenones. Chem. Commun. 53, 6828-6831. doi: 10.1039/C7CC01666B

Conflict of Interest: The authors declare that the research was conducted in the absence of any commercial or financial relationships that could be construed as a potential conflict of interest.

Copyright (c) 2020 Zhang, Hao, Cai, Li, Tu and Jiang. This is an open-access article distributed under the terms of the Creative Commons Attribution License (CC BY). The use, distribution or reproduction in other forums is permitted, provided the original author(s) and the copyright owner(s) are credited and that the original publication in this journal is cited, in accordance with accepted academic practice. No use, distribution or reproduction is permitted which does not comply with these terms. 